\title{
Nucleotide variation and balancing selection at the Ckma gene in Atlantic cod: Analysis with multiple merger coalescent models
}

Einar Árnason, Katrín Halldórsdóttir

High-fecundity organisms, such as Atlantic cod, can withstand substantial natural selection and the entailing genetic load of replacing alleles at a number of loci due to their excess reproductive capacity. High-fecundity organisms may reproduce by sweepstakes leading to highly skewed heavy-tailed offspring distribution. Under such reproduction the Kingman coalescent of binary mergers breaks down and models of multiple merger coalescent are more appropriate. Here we study nucleotide variation at the Ckma (Creatine Kinase Muscle type A) gene in Atlantic cod. The gene shows extreme differentiation between the North (Canada, Greenland, Iceland, Norway, Barents Sea) and the South (Faroe Islands, North-, Baltic-, Celtic-, and Irish Seas) with $F_{S T}>0.8$ between regions whereas neutral loci show no differentiation. This is evidence of natural selection. The protein sequence is conserved by purifying selection whereas silent and non-coding sites show extreme differentiation. The unfolded site- frequency spectrum has three modes, a mode at singleton sites and two high frequency modes at opposite frequencies representing divergent branches of the gene genealogy that is evidence for balancing selection. Analysis with multiple-merger coalescent models can account for the high frequency of singleton sites and indicate reproductive sweepstakes. Coalescent time scales vary with population size and with the inverse of variance in offspring number. Parameter estimates using multiple-merger coalescent models show that times scales are faster than under the Kingman coalescent. 
Nucleotide Variation and Balancing

Selection at the Ckma gene in Atlantic cod: Analysis with multiple merger coalescent models

\author{
Einar Árnason ${ }^{1}$ and Katrín Halldórsdóttir ${ }^{2}$ \\ ${ }^{1}$ Institute of Biology, University of Iceland, Reykjavík, Iceland \\ ${ }^{2}$ Institute of Biology, University of Iceland, Reykjavík, Iceland
}

\title{
\& ABSTRACT
}

High-fecundity organisms, such as Atlantic cod, can withstand substantial natural selection and the entailing genetic load of replacing alleles at a number of loci due to their excess reproductive capacity. High-fecundity organisms may reproduce by sweepstakes leading to highly skewed heavy-tailed offspring distribution. Under such reproduction the Kingman coalescent of binary mergers breaks down and models of multiple merger coalescent are more appropriate. Here we study nucleotide variation at the Ckma (Creatine Kinase Muscle type A) gene in Atlantic cod. The gene shows extreme differentiation between the North (Canada, Greenland, Iceland, Norway, Barents Sea) and the South (Faroe Islands, North-, Baltic-, Celtic-, and Irish Seas) with $F_{S T}>0.8$ between regions whereas neutral loci show no differentiation. This is evidence of natural selection. The protein sequence is conserved by purifying selection whereas silent and non-coding sites show extreme differentiation. The unfolded sitefrequency spectrum has three modes, a mode at singleton sites and two high frequency modes at opposite frequencies representing divergent branches of the gene genealogy that is evidence for balancing selection. Analysis with multiple-merger coalescent models can account for the high frequency of singleton sites and indicate reproductive sweepstakes. Coalescent time scales vary with population size and with the inverse of variance in offspring number. Parameter estimates using multiple-merger coalescent models show that times scales are faster than under the Kingman coalescent.

Keywords: Balancing Selection, Ckma, Atlantic cod, multiple-merger coalescent, time scales

\section{INTRODUCTION}

High fecundity translates into large excess reproductive capacity that would allow organisms to withstand substantial natural selection enabling them to bear the entailing high genetic load. Based on the concept of the cost of natural selection (Haldane, 1957) high-fecundity organisms relative to low-fecundity organisms should at any time be able to adapt a larger proportion of their genome to meet various environmental challenges. Trying to explain the paradox of sexual reproduction Williams (1975) in his Sex and Evolution book argues that high-fecundity coupled with heavy mortality of young (type III survivorship) may be able to pay the $50 \%$ fitness cost of meiosis. He developed 
several models, such as the Elm/Oyster and the Cod/Starfish models, which emphasize the importance of high-fecundity for selection. Williams also discussed the concept of reproductive sweepstakes. There is no heritability of fitness and sexual reproduction continuously assembles Sisyphean genotypes (from Sisyphus who was punished to roll a boulder up a hill only to see it roll back down, and having to repeat his actions forever). The distribution of offspring numbers is highly skewed, heavy-tailed and with high variance (lognormal). That is Williams's fitness distribution. The environment factors are envisioned as acting in a sequence of selective filters. With only a few factors (e.g. temperature, salinity, etc) there nevertheless can be an enormous number of different sequences of selective filters (environments) that do not recur. Hence a winning genotype is not permanent and must be continuously reassembled. Natural selection increases the variance in offspring number and thereby reduces effective population size genome-wide. Neutral variation will therefore drift faster under pervasive natural selection.

Coalescent theory (Kingman, 1982a,b) traces the genealogy of a sample and is very useful for making statistical inferences from molecular population genetic data. However, in an extreme case under a winner-take-all sweepstakes reproduction all samples would coalesce immediately in the previous generation (Árnason, 2004) and there would be no variation. However, this extreme case is not realistic. The Kingman coalescent, which is derived from Wrigth/Fisher models of low fecundity non-skewed offspring distributions, assumes a bifurcating genealogy and is not appropriate for reproduction of this kind (Eldon and Wakeley, 2006; Schweinsberg, 2003; Wakeley, 2013; Tellier and Lemaire, 2014). Some organisms may exhibit both high fecundity and highly skewed offspring distributions. The coalescent for such organisms will lie somewhere between the extreme of a winner-take-all sweepstakes coalescent and the Kingman coalescent. For these organisms the $\Lambda$ coalescent allowing multiple mergers of ancestral lineages at any one generation (Pitman, 1999; Sagitov, 1999; Donnelly and Kurtz, 1999; Eldon and Wakeley, 2006; Schweinsberg, 2003; Sargsyan and Wakeley, 2008 ) or $\Xi$ coalescent allowing simultaneous multiple mergers of ancestral lineages (Schweinsberg, 2000; Möhle and Sagitov, 2001) may be more appropriate. Wakeley (2013) gives an overview of the development of coalescent theory in new directions. There is also active development of statistical inference methods associated with multiple merger coalescents (e.g. Birkner et al., 2013b; Eldon et al., 2015) Studies on the high fecundity organisms Pacific oyster Crassostrea gigas (Hedgecock and Pudovkin, 2011) and Atlantic cod Gadus morhua (Linneaus, 1758) (Árnason and Pálsson, 1996; Árnason et al., 1998, 2000; Carr and Marshall, 1991a; Carr et al., 1995; Pepin and Carr, 1993; Árnason, 2004) have provided data for a number of tests of some of the new coalescent models (Eldon and Wakeley, 2006; Eldon, 2011; Eldon and Degnan, 2012; Steinrücken et al., 2013; Birkner et al., 2013b). A high number of singletons is a feature of sequence studies of high fecundity organisms such as the Atlantic cod. This is expected under models of multiple merger coalescents and, therefore, they perform better than the Kingman coalescent by capturing the high frequency of singletons. Atlantic cod thus provides a model for studies applying the multiple merger coalescent. In this paper we apply some of these new methods for $\Lambda$ coalescents as appropriate neutral null models for high fecundity organisms in a study of balancing selection at a gene showing extreme spatial differentiation in Atlantic cod. 
In general the time scale of multiple merger coalescent models can be much shorter than for a Kingman coalescent. For example, under the $\operatorname{Beta}(2-\alpha, \alpha)$ coalescent model time scales depend on $N^{\alpha-1}$ (Schweinsberg, 2003; Eldon et al., 2015). Under the extreme winner-take-all sweepstakes coalescent mentioned above all individuals would be sibs differing only by new mutations. However, this is an extreme case. Real world multiple merger coalescents lie somewhere between this extreme and the Kingman coalescent. Applying multiple merger coalescents does not imply that we are sampling siblings or that samples from say Greenland and Norway share the same parents. Möhle (1998) and elsewhere shows that for large $N$ the Kingman coalescent is robust because the influence of structure of various types (selfing, age structure, geographic structure) occurs on a shorter time scale than the time scale of the coalescent. Under the Kingman coalescent the expected time to the most recent coalescence of a sample of $n$ individuals is $2 /(n(n-1))\left(\times 2 N_{e}\right.$ generations). Although the general robustness of the Kingman coalescent breaks down under multiple merger coalescents, coalescence times will nevertheless be longer than a single generation. Although time scales under multiple merger coalescents are shorter than under Kingman coalescent, and for example our estimates are square root or cube root of $N_{e}$, they are longer than a single generation of the winner-take-all sweepstakes coalescent.

A dense genomic map of genetic variation in humans (and in model organisms) allows scanning the genome for signatures of natural selection (Voight et al., 2006; Sabeti et al., 2007; Storz, 2005). The density of the genetic maps and sensitivity of the various methods used influences what percentage of the human genome we observe to show footprints of selection (Voight et al., 2006; Sabeti et al., 2007; Storz, 2005). It is safe to say that only a small percentage of single nucleotide polymorphisms (SNPs) show footprints of selection in the low fecundity humans (Akey, 2009; Pickrell1 et al., 2009). For microsatellite loci $2 \%(13 / 624)$ were detected as outliers when African and nonAfrican human populations were compared (Storz et al., 2004). In contrast, comparable genome level studies in Atlantic cod find that 11\% (26 out of 235) of independent SNPs (Moen et al., 2008) are $F_{S T}$ outliers (by method of Beaumont and Nichols, 1996) and $4 \%$ SNPs (70 out of 1641 Bradbury et al., 2010) are Bayscan outliers (by method of Foll and Gaggiotti, 2008) likely undergoing selection. Similarly one fourth of microsatellite loci in Atlantic cod (Nielsen et al., 2006) are $F_{S T}$ outliers. This supports our thesis that a considerable fraction of the Atlantic cod genome may be simultaneously under selection for different adaptations.

More than half of the 70 outliers in Bradbury et al. (2010) study of Atlantic cod show adaptive parallel clines related to temperature on both the western and eastern side of the Atlantic Ocean. They show that multiple genes, located in three independent linkage groups, are involved. There are single genes as well as blocks of genes in "genomic islands" (Bradbury et al., 2013; Hemmer-Hansen et al., 2013). Some of the genes or blocks of genes show clear spatial patterns while other genes show complex spatio-temporal patterns in contrast to no differentiation of non-outlier (neutral) loci (Poulsen et al., 2011; Therkildsen et al., 2013). For example a locality in West Greenland shows great similarity to coastal areas in Iceland, implying either parallel adaptation on a fine scale or patterns of gene flow that are hard to reconcile with geographic distance. Another study (Hemmer-Hansen et al., 2014) adds even more complexity of population structure at outlier loci with little or no difference at non-outlier neutral loci. 
A study of differentiation among four Atlantic cod populations along the coast of Norway (Moen et al., 2008) showed no differentiation among presumably neutral non-outliers loci with an average $\bar{F}_{S T}=0.0012$. In contrast, among the outlier loci, presumably under selection, the $F_{S T}$ ranged from 0.08 to extreme differention of 0.83 with an average $\bar{F}_{S T}=0.27$. Here we analyze in detail nucleotide variation at a large fragment of the Ckma gene (encoding a muscle isoform A of creatine kinase) showing extreme spatial differention (Moen et al., 2008) to understand the nature of selection.

Creatine kinases (CK) are crucially important in bioenergetic processes in cells and tissues (Wallimann et al., 1992, 2011). The creatine kinase/phosphocreatine system $(\mathrm{CK} / \mathrm{PCr})$ is an intracellular energy shuttle. CK generates Phosphocreatine $(\mathrm{PCr})$ at the sites of ATP production in glycolysis and oxidative phosphorilation in mitochondria and regenerates ATP from PCr at subcellular sites of ATP use by ATPases. The physiological advantage is to provide a spatial and temporal energy buffer storing and releasing energy in and from PCr. Importantly the rate of intracellular diffusion of both Creatine $(\mathrm{Cr})$ and PCr is one and three orders of magnitude faster than diffusion of ATP and ADP respectively (see Wallimann et al., 1992, 2011, for a detailed account of the CK/PCr system).

We thus have here a gene with a well defined and well understood function. The gene shows extreme spatial differentiation most likely due to selection considering the contrasting behavior of neutral non-outliers. We apply methods of multiple merger $\Lambda$ coalescents, as a new and appropriate null model of neutrality for organisms with highly skewed heavy-tailed offspring distributions, to nucleotide variation of the gene to better understand the nature of selection.

\section{MATERIALS AND METHODS}

\section{Population sampling}

We randomly sampled 180 individual cod from various localities from the distributional range of Atlantic cod (Figure S1). The samples come from our large sample database of greater than 20,000 individuals. All localities are represented with at least 100 individuals (except the White Sea with 24 individuals). The localities are the waters around Newfoundland (New), Greenland (Gre), Iceland (Ice), Faroe Islands (Far), Norway (Nor), and the Barents Sea, North Sea (Nse), Celtic Sea (Cel), Irish Sea (Iri), Baltic Sea (Bal), and the White Sea (Whi). We took a large sample from Iceland and stratified the sampling to get about 8-10 individuals from the other localities to cover the widest geographic range possible with our database. After cloning, sequencing and quality checking as detailed below we had 122 individals covering a wide geographic area from the Southwest/Northwest to the Northeast and South.

We included samples of the closely related taxa Arctic cod Boreogadus saida (Lepechin, 1774) (Bsa) and Greenland cod G. ogac (Richardson, 1836) (Gog) both sampled in Greenland waters as well as Pacific cod G. macrocephalus (Tilesius, 1810) (Gma) and Walleye pollock Theragra chalcogramma (Pallas, 1811) (Gch) sampled from the Pacific ocean as outgroups. Carr et al. (1999) and Pogson and Mesa (2004) discuss the relationship and biogeography of these taxa. Coulson et al. (2006) provide the most comprehensive account based on mitochondrial genomics. They consider Arctic cod to be an outgroup for all these taxa. Atlantic cod and Walleye pollock are closely related 
taxa and Pacific cod slightly more distant. Pacific cod and Walleye pollock represent two separate but nearly simultaneous invasions of the Pacific. The Atlantic cod vs. Pacific cod split is dated at 4 mya and the Atlantic cod vs. Walleye pollock split is dated at 3.8 mya using conventional rates of mtDNA evolution (see time scales below). Coulson et al. (2006) suggested a nomenclature revision from Theragra chalcogramma to Gadus chalcogrammus (Pallas, 1814) for Walleye pollock that has been accepted by the American Fisheries Society (Page et al., 2013). We follow the new nomenclature hereafter. Greenland cod is a recent reinvasion of Pacific cod into the Arctic and Coulson et al. (2006) consider it to be a subspecies of Pacific cod.

The Icelandic Committee for Welfare of Experimental Animals, Chief Veterinary Office at the Ministry of Agriculture, Reykjavik, Iceland has determined that the research conducted here is not subject to the laws concerning the Welfare of Experimental Animals (The Icelandic Law on Animal Protection, Law 15/1994, last updated with Law 157/2012). DNA was isolated from tissue taken from dead fish on board research vessels. Fish were collected during the yearly surveys of the Icelandic Marine Research Institute. All research plans and sampling of fish, including the ones for the current project, have been evaluated and approved by the Marine Research Institute Board of Directors. Samples were also obtained from dead fish from marine research institutes in Norway, the Netherlands, Canada and the US that were similarly approved by the respective ethics boards. The samples from the US used in this study have been described in Cunningham et al. (2009) and the samples from Norway in Árnason and Pálsson (1996). The samples from Canada consisted of DNA isolated from the samples described in Pogson (2001). The samples from the Netherlands were obtained from the Beam-Trawl-Survey (http://www.wageningenur.nl/en/Expertise-Services/ Research-Institutes/imares/Weblogs/Beam-Trawl-Survey.htm) of the Institute for Marine Resources \& Ecosystem Studies (IMARES), Wageningen University, the Netherlands, which is approved by the IMARES Animal Care Committee and IMARES Board of Directors.

\section{Molecular analysis}

We used sequences associated with the Moen et al. (2008) high $F_{S T}$ SNP's (Gm3660514 with an $F_{S T}=0.83, G m 366-1022$ with an $F_{S T}=0.82$, and $G m 366-1073$ with an $\left.F_{S T}=0.82\right)$ to make probes to search our Atlantic cod BAC library (GAMH, made for us by Amplicon Express, www.amplicon-express.com). We had positive clones 454 sequenced (Microsynth) and obtained a 34,223 bp scaffold containing the gene of interest. From this sequence we generated primers (Table S1) for PCR amplifying a $4000 \mathrm{bp}$ fragment for population studies. Our scaffold largely but not entirely aligned to GeneScaffold 4232 of the Atlantic cod genome sequence (Star et al., 2011) (www.ensemble.org). We confirmed our primers using the Atlantic cod genome sequence. Our BAC library was made from a single individual from Bay of Faxa (Reykjavik) Iceland and the genomic sequence is based on a specimen from the North East Arctic cod in Norway (Star et al., 2011). The conformity of primer sequences between the two specimens from widely separated geographic localities means that the primers should amplify the fragment of interest in individuals taken from widely separate geographic areas. However, it does not preclude the possibility of ascertainment bias for example due to variation in primer binding sites. The amplification primers 
were long (Table S1) which may facilitate annealing in spite of some variation in primer binding site. Samples from all localities were PCR amplified without issue and there were no signs of ascertainment bias in the molecular results.

We Topo-TA cloned fragments into pCR XL-TOPO vector (Invitrogen). We sequenced clones with M13 primers and sequencing primers (Table S1) using BigDye Terminator kit (Applied Biosystems) and performed sequencing on ABI 3100 and ABI3500XL (Applied Biosystems) automated sequencers.

We applied the same methods and sequenced $711 \mathrm{bp}$ of the Hemoglobin $\alpha 2$ (HbA2) locus (Halldórsdóttir and Árnason, 2009a,b; Borza et al., 2009) and 1021 bp of the myoglobin (Myg) locus (Lurman et al., 2007). The previous studies on these genes (Halldórsdóttir and Árnason, 2009a,b; Borza et al., 2009; Lurman et al., 2007) had not found any signs of selection and we, therefore, used them for neutral locus comparisons. The $H b A 2$ data were of 114 Atlantic cod individuals and 13 individuals of various closely related taxa. The $M y g$ data were from 45 Atlantic cod individuals and two individuals of Pacific cod. Other closely related taxa did not amplify for Myg. The HbA2 and Myg individuals covered much the same geographic localities as $C \mathrm{kma}$.

All sequences have been deposited in Genbank with Ckma accession numbers KM624178 - KM624309, HbA2 accession numbers KM624310 - KM624436, and Myg accession numbers KM624437 - KM624483.

\section{Population genetic analysis}

We base called, assembled and edited sequence reads using phred, phrap and consed (Ewing et al., 1998; Ewing and Green, 1998; Gordon et al., 1998). We aligned sequences using muscle (Edgar, 2004), inspected alignments using seaview (version 4) (Gouy et al., 2009) and generated maximum likelihood trees with phyml (Guindon and Gascuel, 2003) under seaview. We used R (R Core Team, 2013) and the ape, pegas, seqinr, ade4, adegenet, and LDheatmap packages (Paradis et al., 2004; Paradis, 2010; Charif and Lobry, 2007; Dray and Dufour, 2007; Jombart and Ahmed, 2011; Shin et al., 2006) and various functions written by us for managing, analyzing, and plotting the data. We used the MLHKA program (Wright and Charlesworth, 2004) for a maximum likelihood HKA test (Hudson et al., 1987) based on the Kingman coalescent.

By PCR amplifying and cloning of fragments polymerase copy errors in the PCR reaction inevitably will be found in clones. The coalescent methods are especially sensitive to singleton variants and errors that would enter into the data as singleton variants should be removed. To remove PCR errors and ensure authenticity of natural variation among individuals we sequenced three clones from each individual. We claim that taking three clones is sufficient to eliminate PCR errors among clones of an individual and yield a consensus sequence of one allele from that individual. We are taking three copies (clones) of two items (chromosomes or alleles $A$ and $a$ ). Any two of three will always be the same allele ( $A$ and $A$ or $a$ and $a$ ). A third clone (order is not important) will be of that same allele with probability $1 / 2$ and of the alternative allele from the other chromosome with probability $1 / 2$. One of the three has probability $1 / 2$ of being different from the two that are the same. In the first case a consensus sequence will be a true consensus of that allele. In the second case a consensus sequence will be a true consensus except at sites where the third clone (alternative allele) matches one of the 
other clones. That is when a naturally occurring site variant or a PCR error in the third clone matches a PCR error in one of the other two clones. This scenario is expected to be a rare event. The effect of such a rare event would be to generate variation that would look like recombination thus, if anything, reducing measures of linkage disequilibrium.

We thus got consensus sequences for a number of individuals. In some cases parts of a clone had low quality sequence. We visually inspected all variant sites using the above mentioned tools. To maximize the number of individuals and the size of the sequenced fragment we struck a balance between number of individuals and quality of sequence. We removed individuals with short sequences and removed individuals that were not covered by three clones. Also we eliminated regions with a phred quality less than 30 . We thus obtained consensus sequences of three clones from each of 122 Atlantic cod and 10 individuals of closely related taxa covering three fragments of the gene (Figure S2) concatenated to give a total sequence of $2500 \mathrm{bp}$.

We analyzed sequence variation for statistics of neutrality and selection using DNAsp (Rozas et al., 2003) and R functions. Site frequency spectra are a most important summary statistics for coalescent analysis of nucleotide data (Wakeley, 2009). We analyzed site frequency spectra using the Kingman coalescent (Kingman, 1982a) and statistical methods developed for multiple merger $\Lambda$ coalescents (Birkner et al., 2013b). We used software from Bjarki Eldon (Birkner et al., 2013b) (http://page. math . tu-berlin.de/ eldon/programs.html to estimate various parameters of the multiple merger $\Lambda$ coalescents. In particular we used the minimum $\ell^{2}$ distance (Birkner et al., 2013b) (sum of squares) to estimate the parameter $\alpha$ of the $\operatorname{Beta}(2-\alpha, \alpha)$ coalescent (Schweinsberg, 2003) and the $\psi$ parameter of the point-mass coalescent (Eldon and Wakeley, 2006). Using these estimates we generated expected site-frequency spectra for the models and compared them to our observed spectra using a likelihood ratio $G$ test with the multiple-merger coalescent models nested within the Kingman coalescent. We also used the overall $\ell_{2}$ distance (square root of $\ell^{2}$ ) to compare the observed and expected site frequency spectrum of the three genes, $C k m a, H b A 2$, and Myg. We used software from Bjarki Eldon to estimate parameters of algebraic $(\mathbf{A}, \gamma)$ and exponential (E, $\beta$ ) growth models (Eldon et al., 2015) and compared the observed site frequency spectra for the three genes to expectations based on these growth models using the $\ell_{2}$ distance.

\section{RESULTS}

\section{Gene and protein}

The Ckma gene encodes creatin kinase muscle isoform A (CKMA). The locus is 3604 base pairs (bp) in GeneScaffold 4232 (coordinates 332764 to 336367, gene name ENSGMOG00000008778 in the cod genome, www . ensemble. org, Star et al. (2011)). The gene has seven exons (Figure S2). Ensemble reports 382 amino acids (aa). However, both genescan (http://genes.mit.edu/GENSCAN.html) and fgenesh (www. softberry.com) predicted 381 aa. The www. ensemble.org sequence adds a Glycine $(\mathrm{G})$ residue in position 323 apparently due to incorrect splicing at the junction of the last two exons.

For mapping the gene the SNP locus cgpGmo-S497 at position $19.5 \mathrm{cM}$ (see Appendix S3 in Supplementary data of Borza et al., 2010) in linkage group CGP16 is found 
in a partial cDNA mRNA sequence (Genbank accession number EX184243) (Hubert et al., 2010; Borza et al., 2010) matching the Ckma gene.

There are seven paralogous genes found in the Atlantic cod genome (www . ensemble. org) encoding mitochondrial, brain and muscle isoforms of Creatine Kinase. The protein sequence of the two alleles $A$ and $B$ in Atlantic cod and of all the closely related taxa studied were of the CKMA isoform (Figure S3). The variation reported is thus from orthologous genes.

\section{Nucleotide variation and divergence}

The variants of $C \mathrm{kma}$ in Atlantic cod fell into two distinct and divergent groups which we refer to as $A$ and $B$ alleles or haplogroups (Figure 1 and Figure S4). They were fixed for a $\mathrm{C}$ vs $\mathrm{T}$ at site 1732 in the concatenated sequence (Table S2). The alleles also differed at 19 additional sites (Figure 2 and Table S2). However, there was variation at these 19 sites that was segregating at a low frequency within one or both alleles which was evidently from recombination.

The divergence of the $A$ and $B$ alleles has arisen after the speciation between Gadus morhua and its Pacific closely related species G. macrocephalus or G. chalcogrammus. The gross, $D_{X Y}$, and net, $D_{a}$, nucleotide divergence (see also Cruickshank and Hahn, 2014) between the $A$ and $B$ alleles was about one half that of the divergence between the closely related taxa (Table S3). The Ckma and $H b A 2$ divergences between the closely related taxa are very similar but the Myg divergence is about twice that (Figure S5 and Table S3). The variance of times to coalescence is large so it is not unexpected to find differences in divergence among genes. There is nothing in the behavior of Myg and $H b A 2$ to indicate deviation from the multiple merger null hypothesis of neutrality. In contrast with the results of Coulson et al. (2006) the maximum likelihood tree for Ckma (Figure 1) and divergence estimates (Table S3) imply that separation of G. chalcogrammus predates the separation of G. macrocephalus and G. morhua. Similarly, the $H b A 2$ locus showed the same pattern that G. chalcogrammus is outside of G. macrocephalus and G. morhua (Figure S6). Unfortunately the Myg locus did not yield sequences for $G$. chalcogrammus.

All summary statistics showed high variation for Ckma (Table 1). In particular nucleotide diversity $\hat{\pi}$ was high relative to the scaled population size $\hat{\theta}_{S}$ resulting in a non-significant Tajima's $\hat{D}$. This was due to the great number of high heterozygosity sites differing between the two alleles (Figure 2 and Table S2). Considering the North and South population and the $A$ and $B$ alleles separately there was much less variation. Although there were several polymorphic sites within both $A$ and $B$ alleles (Figure 2 and Table S2) nucleotide diversity was lower than for the entire sample and the relative difference of $\hat{\pi}$ and $\hat{\theta}_{S}$ for each allele was greater resulting in negative and significant Tajima's $\hat{D}$. The $H b A 2$ gene had a very low haplotype and nucleotide diversity but disparity with $\hat{\theta}_{S}$ gave overall a negative and significant Tajima's $\hat{D}$. In congruence with divergence measures the $M y g$ locus had high haplotype and nucleotide diversity, albeit lower than $C k m a$, but overall a negative and significant Tajima's $\hat{D}$.

There were five non-synonymous changes segregating as singleton sites within Atlantic cod (Table S2 and Table S4). Two of these were also segregating as singletons within B. saida and G. macrocephalus and one other singleton was also found in $G$. macrocephalus. B. saida was fixed for a Glycine (GGT codon) for which the other 
taxa have a Glutamine (CAG codon) with changes in all three sites of the respective codon (aa number 242). Assuming independent mutations and depending on the path of evolution of that particular codon all three changes may have been non-synonymous.

There was considerable linkage disequilibrium (measured as $D^{\prime}$ ) throughout the gene (Figure S7 and Figure S8). Linkage disequilibrium measures are sensitive to allele frequency (Hedrick, 1987) and in general there is no measure that is independent of allele frequencies (Lewontin, 1988). In Figure S8 we have therefore excluded singleton sites because they will always show maximum linkage disequilibrium. However, low frequency sites generate noise so the signal of linkage disequilibrium is hard to see. We therefore used sites with minor allele frequency greater than an arbitrary frequency of 0.1 (Figure S7) which includes all the intermediate allele frequency (high heterozygosity) polymorphisms and gets rid of low frequency variants that generate noise in the linkage disequilibrium plots. The high frequency sites gave the clearest sign of two blocks of sites with almost full linkage disequilibrium both among sites within and between the blocks. The two blocks are separated by a site of recombination (site 691 in Table S2). On both the $A$ and $B$ allele backgrounds both the ancient $c$ major allele and the derived $t$ minor allele at site 691 were geographically widespread (the $t$ on an $A$ allele background was found in individuals from the Baltic and from Iceland and the $c$ on the $B$ allele background was found in individuals from throughout the North ranging from the White Sea, Barents Sea, Norway, Iceland, Greenland, and Canada).

Two other sites (site three and 12 in Figure S7 that are sites 578 and 1444 in Table S2) showed slight reduction in linkage disequilibrium (Figure S7) and therefore some signs of recombination. Other sites (such as sites 509, 660, 1075 in Table S2) also showed some evidence of recombination. In all these cases the recombinant gametic types with respect to the $A$ and $B$ allelic backgrounds were geographically widespread in general agreements with the result above for site 691.

The results of a maximum likelihood HKA test of selection that is based on the Kingman coalescent (Wright and Charlesworth, 2004) gave a selection parameter $k=$ 2.12 in the direction of balancing selection (Table S5). However, the results were not statistically significant possibly because of too high variation among the presumed neutral loci (HbA2 and $M y g$ ) used for comparison in the test.

\section{Spatial differentiation}

The variation of the Ckma gene was spatially patterned. The $A$ allele was overall at a high frequency of $97 \%$ in an area that we call South (Faroe Islands, North Sea, Baltic Sea, Celtic Sea and Irish Sea) (Table S6). Conversely the $B$ allele was at a high frequency of $92 \%$ in an area that we call North ranging from the Northwest (Nova Scotia and Newfoundland in Canada) through Greenland, Iceland, Norway, Barents Sea and the White Sea. There was variation among localites within each region with some localities having zero frequency presumably due to low sample sizes. We do not have genotypic data and cannot test for Hardy-Weinberg equilibrium. The differentiation of North and South was evident in interlocality $F_{S T}$ values (Table S7) and an overall $F_{S T}=0.763$ between North and South. There was no significant differentiation among localities within either the North or the South but very high and significant differentiation between North and South localities. Similarly, there was great differentiation between the $A$ and $B$ alleles with an $F_{S T}=0.804$. This was in stark contrast to the lack of differentiation 
between North and South at the HbA2 ( $\left.F_{S T}=0.004\right)$ and $M y g\left(F_{S T}=-0.029\right)$ loci.

The high differentiation was mostly due to the great number of high heterozygosity sites differing between the two alleles (Figure 2 and Table S2). Three of the sites were the SNPs already found by Moen et al. (2008) with an $F_{S T}=0.82$ for north and south localities along the coast of Norway. The high frequency sites showed indications of recombination between the $A$ and $B$ alleles (see for example patterns of segregating sites for individuals 105698, 124401, 105657, 200500, 118129, 119535, 118147, and 106620 in Table S2).

There were also several high heterozygosity polymorphic sites within both the $A$ and $B$ alleles (Figure 2). This variation, however, did not show geographical patterns (Table S2). For example sites 1050 and 1428 mutated relative to outgroup within the $A$ alleles were found among individuals from Iceland, White Sea, Celtic Sea, Faroe Islands and the Baltic. Similarly within the $B$ alleles high heterozygosity sites $656,691,1340$, and 1444, which were mutated relative to the outgroup, were all widespread among North localities ranging from the Northwest to the Northeast Atlantic (Figure S1).

\section{Site frequency spectra}

The unfolded site frequency spectrum for the Ckma gene was trimodal (Figure 3), with a mode at singleton sites, a mode at 43 , and a mode at 79 . The latter modes were at opposite frequencies out of a total of 122 and represented the $A$ and $B$ lineages of the genealogy. The Kingman coalescent did not fit the data well. Both the Beta $(2-\alpha, \alpha)$ and point-mass coalescent models gave a much better fit (Table S8) in particular by capturing the singleton class. None of the coalescent models captured the modes at 43 and 79.

In contrast the site frequency spectra for the $H b A 2$ and $M y g$ genes were L shaped with a high peak at singleton sites (Figure S9 and Figure S10). Again the Kingman coalescent did not fit well but both multiple merger coalescent models captured the high frequency of singleton sites.

The site frequency spectra of the $A$ and $B$ alleles alone were bimodal with a high singleton class and peaks around 40 and 78 respectively (Figure S11). The high frequency modes for the two alleles, at 40 and 78 respectively, resulted because most of the high frequency and high heterozygosity sites that separate the two alleles were not fixed within each allele presumably due to recombination between the alleles (Table S2, and see examples presented above).

\section{Coalescent parameter estimates}

Following Birkner et al. (2013b) we used the $\ell^{2}$ distance, the sum of the squared differences between the observed and expected site frequency spectrum (scaled with the number of segregating sites), for estimating parameters of two $\Lambda$ coalescent models, $\hat{\alpha}$ for the $\operatorname{Beta}(2-\alpha, \alpha)$ and $\hat{\psi}$ for the point-mass coalescent (Table 2, Figure S12 and Figure S13). The Kingman coalescent, a null model for which $\alpha=2.0$, had the highest $\ell^{2}$ indicating worst fit among the models. The HbA2 and Myg loci had an $\hat{\alpha}=1.00$ and a $\hat{\psi}=0.23$. The $C k m a$ locus had overall a considerably higher $\alpha$ and lower $\psi$. The parameter estimates for the Ckma alleles separately were similar to those of the presumed neutral loci HbA2 and Myg.

For comparison we also estimated the parameters for the entire dataset of mtDNA 
variation in the North Atlantic (Árnason, 2004) and the various subsamples making up that total sample using the unfolded site frequency spectrum with G. macrocephalus as the outgroup (Table 2 and Figure S13). Previously these have been analysed using the folded site frequency spectrum (see for example Birkner et al., 2013b; Steinrücken et al., 2013). For the total sample, spanning a similar geographic range as the nuclear genes, the parameter estimates differed from the nuclear loci with $\hat{\alpha}=1.53$ and $\hat{\psi}=0.01$. The large samples from Newfoundland and Iceland and the sample from the Faroe Islands gave similar values. The values for Greenland, Norway, White Sea, and Baltic Sea were much closer to the results for the Kingman coalescent $(\alpha=2.0)$. For these localities homoplasies were more frequent in the data than for the total and the large samples. Homoplasies will reduce the number of singletons and move such sites towards the right tail of the site frequency distribution. This explains the higher values for these localities.

\section{Models of multiple merger coalescents and population growth}

It is important to see how a locus deviates from a null model of neutrality to understand selection. Here the null model is multiple merger $\Lambda$ coalescents instead of the Kingman coalescent. Following Birkner et al. (2013b) we used the $\ell_{2}$ distance, the square root of the sum of the squared differences between the observed and expected site frequency spectrum. The overall $\ell_{2}$ distance for the three loci between the observed site frequency spectrum and expectations based on the two $\Lambda$ coalescent models are in Table S9. The Ckma had the highest overall distance (the worst fit). There is clearly something special about the Ckma locus that was not seen among the other loci. In particular the trimodal site frequency spectrum is a sign of natural selection. We did not see these for the other genes. Admittedly this is not a formal test of selection. But Ckma behaved differently. This is a locus specific behavior that is most likely a sign of selection.

The high frequency of singletons is predicted both by population growth and by $\Lambda$ and $\Xi$ multiple merger coalescents. Eldon et al. (2015) find that the weight of the right tail of the site frequency spectrum may have features allowing one to distinguish between population growth and $\Lambda$ coalescents. Eldon et al. (2015) have developed methods for such analysis which we apply here. Using both the $\ell_{2}$ distance and approximate log likelihood we find (Table S10) that the algebraic $(\mathbf{A}, \gamma)$ and exponential $(\mathbf{E}, \beta)$ growth models gave very similar fits for each of the three genes. Again, as with the multiple merger coalescent models (Table S11), the Ckma gene stood out and had the worst fit. The Myg gene showed equally good fit to the the two growth models and the $\operatorname{Beta}(2-\alpha, \alpha)$ coalescent model. For both the $C k m a$ and $H b A 2$ genes the growth models showed worse fit than the coalescent models. However, this comparison of $\ell_{2}$ distances does not constitute a formal test as stated above.

\section{DISCUSSION}

\section{Genes and proteins}

The CKMA protein is highly conserved among the investigated taxa. The single aa difference between $B$. saida and the other species presumably is adaptive with all sites of the codon having changed. The few aa variants were all singletons in the sample. In fact most of the variation is in non-coding regions and all the high heterozygosity sites in coding regions are synonymous changes. Given the high conservation of the protein and 
the high variation among silent and non-coding sites that are indicative of the mutational pressure the singleton non-synonymous changes are likely slightly deleterious and will be removed by purifying selection. Some or even all of the silent and non-coding differences between the $A$ and $B$ alleles may be functional control elements important in expression in different tissues or under different environments. The potential functional differences remain to be studied.

The HbA2 and Myg genes have well defined functions. They are probably under purifying selection. They were taken as independent genes in separate linkage groups for comparison. A caveat is that genetic variation at unlinked sites may be correlated and not independent in high fecundity populations with skewed distribution of offspring (Eldon and Wakeley, 2008; Birkner et al., 2013a). The question remains, however, whether and to what extent such dependence impacts inference.

\section{Three hypotheses}

We discuss three possible explanations for the observed patterns of great divergence of the $A$ alleles and $B$ alleles, their spatial differentiation, and the trimodal site-frequency spectrum. The first explanation is the isolation/admixture hypothesis, the second is the $\Xi$ coalescent of simultaneous multiple mergers in any one generation, and the third is the balancing selection hypothesis. Our interpretation is that the evidence favors balancing selection.

\section{Ancient isolation and recent admixture}

First, there is the possibility of recent admixture of anciently separated and divergent gene pools that have come together in a hybrid zone of secondary contact (Bowcock et al., 1991; Bernardi et al., 1993; Guinand et al., 2004). The spatial patterns of genetic separation between the South (Faroe Islands, North Sea, Baltic Sea, Celtic Sea and Irish Sea) and the North (Nova Scotia and Newfoundland, Greenland, Iceland, Norway, Barents Sea, and White Sea) could be taken as evidence for this. The South is a shallow water environment whereas the the North has more diversity of depth ranging from shallow to deep waters. Differences in temperature, salinty and other environmental factors are correlated with the North South difference. The great nucleotide divergence between the North and the South would imply either that this is an ancient divergence (not a Pleistocene event) or even a not-so-ancient divergence driven by strong selection over a shorter time. If the time of separation of G. morhua and G. macrocephalus and $G$. chalcogrammus is taken at 3.8-4.0 Mya (Coulson et al., 2006) the time of separation of the $A$ and $B$ clades would then be 2 Mya based on the nucleotide divergence of the $A$ and $B$ clades which we show is one half that of the closely related taxa. An even lower divergence time of 2.1 Mya has been suggested (Pogson and Mesa, 2004) that would still leave the divergence of the $A$ and $B$ clade at 1 Mya. These divergence times, however, are all based on the Kingman coalescent and the faster time scales of the multiple merger coalescent are discussed below.

A counter argument is that isolation and admixture are part of the breeding structure of a population leaving genome-wide impacts (Wright, 1931). Therefore, different genes should be concordant in their behavior (Bernardi et al., 1993). This should be true for neutral genes that randomly drift apart in the different isolated areas. Genes under selection adapting to the different environments of the isolated areas should show even 
greater divergence. The $H b A 2$ and the $M y g$ show no differentiation between the North and the South. Also the non-outlier SNPs in Moen et al. (2008) show no differentiation whereas three SNPs of the $C k m a$ gene show high and extreme $F_{S T}$. The correspondence between our results and those of Moen et al. (2008), with very similar $F_{S T}$ between our North vs. South and the north vs. south along the coast of Norway in Moen et al. (2008) is strong independent verification of our main result. The Ckma was after all the most extreme outlier in Moen et al. (2008). Similarly, Bradbury et al. (2010) find that non-outlier SNPs show no differentiation although other SNPs show differentiation from parallel adaptation to temperature on the eastern and western side of the Atlantic Ocean. Nielsen et al. (2003) describe a pattern of microsatellite variation in a transition area between the Baltic and Danish Belt Sea which they interpret as a hybrid zone. There is no evidence for a hybrid zone at that location in the Ckma data. In fact, specific variants within the $A$ allele are widely distributed among localities in the South including the Baltic Sea. This implies gene flow among localities in the South. Similar patterns within $B$ alleles imply gene flow among localities in the North. If indeed there is a hybrid zone for the Ckma gene it would lie between the Faroe Islands on one hand and Iceland and north and middle Norway on the other hand. Considering the North East Arctic and Coastal cod in Norway as an admixture of isolated populations (Pogson and Fevolden, 2003; Árnason and Pálsson, 1996) would add a third hybrid zone within the distribution of the species. It is not a parsimonious explanation to consider there to be multiple hybrid zones of secondary contact within distribution of the species.

For comparison one can consider the Pan I locus (Fevolden and Pogson, 1995, 1997) that clearly is under selection (Pogson, 2001; Pogson and Mesa, 2004) related to depth and fisheries (Sarvas and Fevolden, 2005; Case et al., 2005; Árnason et al., 2009). At face value the locus shows similar differentiation between North and South (Sarvas and Fevolden, 2005) as the Ckma locus. However, the details differ and the parallels between the Pan I and Ckma genes are more apparent than real. Pogson and Fevolden (2003) argue that specific neutral alleles found within a functional class (the Pan I A allel) should show differences between historically isolated regions. Under the historical (isolation/admixture) hypothesis different neutral alleles will drift to high frequencies or fixation in geographic regions isolated from each other. Under the selection hypothesis they should move seamlessly among localities within the putative isolated regions. Pogson and Fevolden (2003) tested the "historical" and "selection" hypotheses (c.f. Árnason and Pálsson, 1996) of Atlantic cod in northern Norway by studying presumed neutral variation among the PanI $A$ alleles in coastal and Arctic localities. In short they found no evidence supporting the historical hypothesis. In fact there were greater differences among coastal localities and between the two Arctic localites than overall between the Arctic and coastal areas. Because of the heterogeneity among coastal localities Pogson and Fevolden (2003) also rejected the selection hypothesis because neutral mutations would move freely among localities within a region and should not show any structure. However, under a skewed offspring distribution and sweepstakes reproduction there can be substanital population structure as measured by $F_{S T}$ in the face of considerable gene flow (Eldon and Wakeley, 2009). Thus their results do not seem at odds with a multiple merger coalescent model.

For Pan I the $B$ allele is largely absent in the South. But the absence of an allele from a certain region cannot be used as evidence for the isolation of populations from 
that region from populations in other regions. Instead under the isolation/admixture (historical) hypothesis one would expect (Pogson and Fevolden, 2003) specific Pan I A alleles to be present characterizing the South and another set of $A$ alleles characterizing the North. But that is not the case; among the various $A$ alleles there is no specific clade of Pan I $A$ alleles in the South (Hernandez and Árnason unpublished). However, for the $C k m a$ gene there is a specific allele, namely the $A$ allele, that is at a high frequency and characterizes the South.

The Pan I $B$ allele which is adapted to the deep (Pampoulie et al., 2007; Árnason et al., 2009) is largely absent from the South. . The Pan I $B$ allele, which is found in the North and in deep water, is much less variable than the Pan I A alleles (Pogson, 2001). This is opposite to what we find for the Ckma A alleles (the South allele) which has less variation than the $C k m a B$ allele (Figure 1) although this is not seen in the summary statistics (Table 1) because of greater recombinational variation at the base of the $A$ clade (Table S2). Also the Pan I locus variation is more related to depth than to geography (Árnason et al., 2009). Under the admixture hypothesis these two loci and all loci showing genome wide effects are expected to show the same pattern.

Under the isolation/admixture hypothesis one would expect recombinant types to be restricted geographically to the zone of secondary contact. This was not the case. We, therefore, think it is more likely that the two blocks of nucleotide sites are held together in linkage disequilibrium by epistatic fitness interactions and that there has been a build up of linkage disequilibrium over time.

Overall, therefore, we find that the Ckma gene does not fit the hypothesis of ancient divergence of gene pools and admixture in secondary contact.

\section{$\Xi$ colaescent and site frequency spectra}

The trimodal site frequency spectrum is not predicted by any of the coalescent models considered here, the Kingman coalescent and the two $\Lambda$ coalescent models, the $\operatorname{Beta}(2-\alpha, \alpha)$ (Schweinsberg, 2003) and the point-mass coalescent (Eldon and Wakeley, 2006). Under the $\Lambda$ coalescent at most a single multiple merger event occurs at any one time. The distribution of family size is of interest and the parameter $\alpha$ influences the probability of getting large families. Under the $\operatorname{Beta}(2-\alpha, \alpha)$ coalescent model the probability of a family size of $k$ or more viable offspring decays like $k^{-\alpha}$ (Schweinsberg, 2003) in the limit of a large $k$. The pool of viable offspring is then resampled to form the next generation under the same conditions. For the Kingman coalescent $\alpha \geq 2$ and there is little chance of seeing large families. For the $\operatorname{Beta}(2-\alpha, \alpha)$ coalescent $1 \leq \alpha<2$ and the lower $\alpha$ the greater is the chance of seeing a large family (Schweinsberg, 2003). The $\psi$ parameter of the point-mass coalescent (Eldon and Wakeley, 2006) similarly measures the proportion of the population that is the offspring of a single individual and is thus an indicator of reproductive sweepstakes. Our estimates of $\psi$ indicate reproductive sweepstakes at the neutral loci and within the $A$ and $B$ alleles of $C k m a$. Balancing selection at $C \mathrm{kma}$ lessens the effects of sweepstakes reproduction. Sweepstakes reproduction has been detected in other high fecundity organisms (Hedgecock and Pudovkin, 2011; Harrang et al., 2013).

Under the more general $\Xi$ coalescent $0<\alpha<1$ (Schweinsberg, 2000) there can be many large families independently in each generation. It would seem that this process could generate multimodal site frequency spectra. Indeed in simulations of $\Xi$ 


\section{PeerJ Reviewing Manuscript}

coalescence site frequency spectra can display multiple modes (Bjarki Eldon personal communication). This question needs further theoretical work. In terms of the concept of sweepstakes reproduction multiple local sweepstakes could have this effect on the site frequency spectrum. Under local sweepstakes genetic structure may be ephemeral (Johnson and Wernham, 1999). Whether this affects the location of the modes and the exact shape of the site frequency spectrum under $\Xi$ coalescent is not known. However, one would not expect build-up of sites around a specific mode of the site frequency spectrum or of two modes at opposite frequencies as at $\mathrm{Ckma}$. Also there should be no particular or regular geographical pattern. We, therefore, think that bumps in the site frequency spectrum under $\Xi$ coalescent is not a good explanation for the $C \mathrm{kma}$ spectrum.

Models of population growth can account for the high frequency of singletons. However, these models also do not predict the trimodal site frequency spectrum observed at Ckma. This is a locus specific behavior that is most likely due to balancing selection.

It is of course possible that population growth and sweepstakes could be occurring at the same time. We do not at this time have methods that estimate simultaneous multiple merger coalescents and population growth and evaluate the relative contribution of each. It is likely that disentangling the effects changes in population size and sweepstakes reproduction may be hard. For example Birkner et al. (2009) discuss how recurrent bottlenecks may construct simultaneous multiple merger $\Xi$ coalescent.

\section{Balancing selection}

Balancing selection generates long branches in the genealogy and neutral variation accumulates on the branches. The balanced functional types (the Ckma $A$ and $B$ alleles in this case) act as they were separate and isolated populations accumulating neutral variation. Recombination can bring variation from one branch to another acting like migration that brings alleles from one population to another (Charlesworth et al., 1997, 2003; Charlesworth, 2006). However, the molecular signatures of balancing selection depend on many factors. Is it a long standing, even trans-species, polymorphism such as MHC in human and chimpanzee (Fan et al., 1989; Nei and Hughes, 1991)) or is it very recent? Examples of the latter are human glucose 6 phosphate dehydrogenase (G6PD) (Verrelli et al., 2002), and hemoglobin $\beta S$ (Currat et al., 2002) and hemoglobin $\beta E$ (Ohashi et al., 2004) and spatially divergent selection of lactase persistence (Tishkoff et al., 2007; Ranciaro et al., 2014) in which a particular allele sweeps a chromosomal segment to an intermediate equilibrium frequency. In these instances recombination has not had time to break up linkage disequilibrium which can extend over large regions. There is very little variation among the new alleles while the alternative chromosomes show much more variation in this region representing the standing variation in the population at the start of the partial sweep.

The effects of a long standing single locus balancing selection will extend only short distances with free recombination and will be difficult to detect (Wiuf and Hein, 1999). If, however, there are obvious signs of a long standing balanced polymorphism it is likely due to a build-up of co-adapted complexes of epistatic interactions among multiple sites and/or suppression of recombination (Wiuf and Hein, 1999). The concept of a supergene of multiple co-adapted sites possibly locked together by structural variation (Thompson and Jiggins, 2014) such as found in butterfly mimicry (Joron et al., 2011) is relevant. 


\section{PeerJ Reviewing Manuscript}

There also can be both partial and complete selective sweeps of new types within each allele of a supergene. Such intra-allelic selective sweeps would reduce variation within and increase variation between alleles. Such reduction of variation could look similar to that for a recent balanced polymorphism except that it would not be limited to one functional type. Thus Pogson (2001) argues that he has detected on-going partial sweeps within each of the two Pan I alleles of Atlantic cod.

Pogson and Mesa (2004) further argue that the Pan I polymorphism is older than speciation of Atlantic cod and Walleye pollock, the closest relatives. The Pan I locus is in a "genomic island" (Bradbury et al., 2013; Hemmer-Hansen et al., 2013) a potential supergene of co-adapted complexes possibly locked together by structural variation. Hernandez and Árnason (unpublished) find large number of differences between the two functional Pan I types in a $12.5 \mathrm{~kb}$ region around the PanI gene that are too extensive to be a partial sweep of a new allele. Such variation is likely to have built up over some time by selection (see time scales below). This is in face of considerable gene flow implied by lack of differentiation of neutral loci (Moen et al., 2009; Bradbury et al., 2010; Eiríksson and Árnason, 2013; Hemmer-Hansen et al., 2014). Similarly, the wide distribution of variants within both the $A$ and $B$ alleles of $C k m a$ implies gene flow among localities within South and within North areas. The recombinant haplotypes between the $A$ and $B$ alleles of $C k m a$ imply gene flow between the South and the North localities.

The observation that the amino acid sequences are conserved might be taken as evidence that there is only purifying selection at the locus. However, claiming balancing selection does not necessarily imply amino acid differences. There is evidence for positive selection in non-coding DNA in other systems (e.g. Drosophila, Andolfatto, 2005) and methods have been developed to detect positive and balancing selection in non-coding regions (e.g. Zhen and Andolfatto, 2012). Balancing selection has also been detected in regulatory regions in other systems. For example, the $5^{\prime}$ cis regulatory region of CCR5 shows evidence for balancing selection (Bamshad et al., 2002), the promoter region of the human Interleukin 10 gene (Wilson et al., 2006), a regulatory region upstream from the human $U G T 2 B 4$ gene (Sun et al., 2011), the NE1 locus in modern Humans and Neanderthals (Gokcumen et al., 2013), and in the 5' UTR's of upregulated genes and genes for effector proteins of a plant-pathogenic fungus (Rech et al., 2014). We have not identified a specific target of selection and we speculate that there is selection on regulatory regions $\left(5^{\prime}, 3^{\prime}\right.$, intronic, and even silent sites that may influence regulation) of the $C \mathrm{kma}$ gene.

$C k m a$ had the highest $F_{S T}$ among all loci studied by Moen et al. (2008) and, therefore, the focus of selectionis likely either the gene itself or a very tightly linked locus. We have looked in www. ensmeble.org what genes are in the close neighborhood. There are no obvious candidates among them for a gene under strong selection. We think, however, that an answer to this question must await a more detailed analysis of a larger region around the Ckma gene.

The Kingman and multiple-merger $\Lambda$ coalescent models that we apply here are models of neutrality. One could argue that it is not appropriate to apply such neutral models to the Ckma locus that is already suspected to be under selection. However, understanding how the locus deviates from neutrality is important for understanding the pattern of selection. Under the neutral theory (Kimura, 1983) polymorphism within species is the transient phase of molecular evolution that leads to divergence between 
species. This is the rational for the HKA test of selection or neutrality (Hudson et al., 1987) that neutrally evolving genomic regions should have the same proportion of polymorphism to divergence, Balancing selection would tend to increase the level of polymorphism within species relative to divergence between them. The results of HKA test are in the direction of balancing selection. The HKA test shows a relative slowing down of divergence to rate of polymorphism at the Ckma locus.

Similarly we consider the peaks in the site frequency spectrum of the Ckma gene to be evidence for balancing selection. The trimodal site frequency spectrum with two high frequency peaks at opposite frequencies that fold into one peak in a folded site frequency spectrum points to the build-up of variation over time. Under a recent balanced polymorphism scenario, such as G6PD and $\beta$ globins in humans, there would be one peak at a particular frequency in the site frequency spectrum representing all sites at which the new allele differs from the ancient alleles. There could be multiple peaks representing high frequency polymorphisms among the ancient alleles. However, they are not expected to be at opposite frequencies to the frequency of the new allele. We, therefore, argue that the pattern at Ckma represents a balanced polymorphism that has been built up over time.

\section{Coalescent parameter estimates and time scales}

The question of coalescent time scale, however, must be considered. Under the Kingman coalescent time is measured in terms of $N / \sigma^{2}$, population size scaled by the variance of family size (Sagitov, 1999; Árnason, 2004; Tavaré, 2004). With a Poisson distribution of family size $\sigma^{2}=1$ for a constant size haploid population and, therefore, time scales with $N$ under the Kingman coalescent. In an extreme winner-take-all sweepstakes $\sigma^{2}=N$ and a sample would coalesce in the previous generation and there would be no variation (Árnason, 2004). In more realistic multiple merger coalescent models the time scale is the quantity $c_{N}=\frac{E\left(v_{1}-1\right)^{2}}{N-1}$ where $c_{N}$ is the probability of two lineages coalescing in the previous generation in a haploid population of fixed size $N$ and $v_{1}$ is the random number of offspring of individual 1 (Sagitov, 1999). In general the time scale of multiple merger coalescent models can be much shorter than for Kingman coalescent. Under the $\operatorname{Beta}(2-\alpha, \alpha)$ coalescent model time scales with $N^{\alpha-1}$ (Schweinsberg, 2003; Eldon et al., 2015). For this model our estimates of $\alpha$ for the nuclear genes are quite low which implies very short time scales. The neutral genes would seem to coalesce in the very recent past. The $A$ and $B$ alleles of $C k m a$ run on very similar time scales to the neutral genes and the locus itself at a slower rate due to the balancing selection with a time scale approximately the cube root of the effective population size $N_{e}$. The mitochondrial DNA runs at yet another and slower time scale. For mtDNA time scales with approximately the square root of $N$. Predicted turnover of alleles is faster and ages of alleles shorter under multiple merger coalescent (Eldon, 2012). Different populations and species may run on different time scales (Eldon and Degnan, 2012) complicating divergence time estimates. Estimates based on Kingman coalescent of divergence times of Atlantic cod populations (Bigg et al., 2008) or divergence of gadid taxa (Coulson et al., 2006) may therefore be too high and may need revision. 


\section{PeerJ Reviewing Manuscript}

\section{Conclusion}

The Ckma protein coding sequence is conserved between all but the most distantly related Arctic cod. The amino acid variants are all singletons in the sample. Based on these facts we conclude that the protein coding sequence is under purifying selection. At the same time silent and non-coding variation at the locus shows extreme spatial differentiation with an $F_{S T}$ greater than 0.8 between the North and the South regions. The regulatory function of this variation is unclear. We argue that the high and locusspecific $F_{S T}$, the highest seen so far for any locus and any spatial comparison in Atlantic cod, indicates that selection and not admixture of anciently divergent gene pools is responsible. Selection is likely to be very strong. It follows that $C \mathrm{kma}$ (or an extremely tightly linked locus) is the focus of selection because the highest $F_{S T}$ indicates the site of action of selection (Nielsen, 2005). Some of the variation may be neutral having risen in frequency within the balanced functional allele where it arose (Charlesworth, 2006). Alternatively some of the variation may be due to selection building co-adapted complexes (Thompson and Jiggins, 2014). In addition to a high peak at singleton sites, higher than that predicted by the Kingman coalescent and characteristic of the multiple-merger coalescent, the site frequency spectrum has two high-frequency modes at opposite but matching frequencies representing the two branches of the genealogy. This pattern is further support for balancing selection. Our estimates of parameters of multiple-merger $\Lambda$ coalescent show that time-scales are fast in accordance with theoretical expectations.

\section{ACKNOWLEDGMENTS}

We thank Jarle Mork (Norwegian University of Science and Technology), Kristján Kristjánsson (Marine Research Institute in Reykjavik), Grant Pogson (University of California at Santa Cruz), Remment ter Hofstede (Institute for Marine Resources and Ecosystem Studies in the Netherlands), and Michael Canino (National Oceanic and Atmospheric Administration) for help in securing some of the samples. We thank Brenda Ciervo Adarna, Guðni Magnús Eiríksson, Lilja Stefánsdóttir, Ragnheiður Fossdal, Svava Ingimarsdóttir, Ubaldo Benitez Hernandez for help with some of the laboratory work. We thank Bjarki Eldon for programs and help with coalescent parameter estimation and for critical comments on the manuscript. We thank R.C. Lewontin for discussions and critical comments on the manuscript. Funding was provided by Icelandic Science Foundation grant of excellence (nr. 40303011), a University of Iceland Research Fund grant, and a SA Private Foundation grant to Einar Árnason and a doctoral grant from the University of Iceland Research Fund to Katrín Halldórsdóttir. 


\section{LITERATURE CITED}

Akey, J. (2009). Constructing genomic maps of positive selection in humans: Where do we go from here? Genome Research, 19:711-722.

Andolfatto, P. (2005). Adaptive evolution of non-coding DNA in Drosophila. Nature, 437:1149-1152.

Árnason, E. (2004). Mitochondrial cytochrome $b$ DNA variation in the high-fecundity Atlantic cod: Trans-Atlantic clines and shallow gene genealogy. Genetics, 166:18711885.

Árnason, E., Hernandez, U. B., and Kristinsson, K. (2009). Intense habitat-specific fisheries-induced selection at the molecular Pan I locus predicts imminent collapse of a major cod fishery. PLoS ONE, 4:e5529.

Árnason, E. and Pálsson, S. (1996). Mitochondrial cytochrome $b$ DNA sequence variation of Atlantic cod, Gadus morhua, from Norway. Molecular Ecology, 5:715724.

Árnason, E., Pálsson, S., and Petersen, P. H. (1998). Mitochondrial cytochrome $b$ DNA sequence variation of Atlantic cod, Gadus morhua, from the Baltic- and the White Seas. Hereditas, 129:37-43.

Árnason, E., Petersen, P. H., Kristinsson, K., Sigurgíslason, H., and Pálsson, S. (2000). Mitochondrial cytochrome $b$ DNA sequence variation of Atlantic cod from Iceland and Greenland. Journal of Fish Biology, 56:409-430.

Bamshad, M. J., Mummidi, S., Gonzalez, E., Ahuja, S. S., Dunn, D. M., Watkins, W. S., Wooding, S., Stone, A. C., Jorde, L. B., Weiss, R. B., and Ahuja, S. K. (2002). A strong signature of balancing selection in the $5^{\prime}$ cis-regulatory region of CCR5. Proceedings of the National Academy of Sciences, 99:10539-10544.

Beaumont, M. A. and Nichols, R. A. (1996). Evaluating loci for use in the genetic analysis of population structure. Proceedings of the Royal Society, B., 263:1619-1626.

Bernardi, G., Sordino, P., and Powers, D. A. (1993). Concordant mitochondrial and nuclear DNA phylogenies for populations of the teleost fish Fundulus heteroclitus. Proceedings of the National Academy of Sciences, 90:9271-9274.

Bigg, G. R., Cunningham, C. W., Ottersen, G., Pogson, G. H., Wadley, M. R., and Williamson, P. (2008). Ice-age survival of Atlantic cod: agreement between palaeoecology models and genetics. Proceedings of the Royal Society, B., 275:163-172.

Birkner, M., Blath, J., and Eldon, B. (2013a). An ancestral recombination graph for diploid populations with skewed offspring distribution. Genetics, 193:255-290.

Birkner, M., Blath, J., and Eldon, B. (2013b). Statistical properties of the site-frequency spectrum associated with $\Lambda$-coalescents. Genetics, 195:1037-1053.

Birkner, M., Blath, J., Möhle, M., Steinrücken, M., and Tams, J. (2009). A modified lookdown construction for the Xi-Fleming-Viot process with mutation and populations with recurrent bottlenecks. Alea, 6:25-61.

Borza, T., Higgins, B., Simpson, G., and Bowman, S. (2010). Integrating the markers Pan I and Haemoglobin with the genetic linkage map of Atlantic cod (Gadus morhua). BMC Research Notes, 3:261.

Borza, T., Stone, C., Gamperl, A. K., and Bowman, S. (2009). Atlantic cod (Gadus morhua) hemoglobin genes: multiplicity and polymorphism. BMC Genetics, 10:51.

Bowcock, A. M., Kidd, J. R., Mountain, J. L., Herbert, J. M., Carotenuto, L., Kidd, K. K., 


\section{PeerJ Reviewing Manuscript}

and Cavalli-Sforza, L. (1991). Drift, admixture, and selection in human evolution: A study with DNA polymorphisms. Proceedings of the National Academy of Sciences, 88:839-843.

Bradbury, I. R., Hubert, S., Higgins, B., Borza, T., Bowman, S., Paterson, I. G., Snelgrove, P. V. R., Morris, C. J., Gregory, R. S., Hardie, D. C., Hutchings, J. A., Ruzzante, D. E., Taggart, C. T., and Bentzen, P. (2010). Parallel adaptive evolution of Atlantic cod on both sides of the Atlantic Ocean in response to temperature. Proceedings of the Royal Society, B., 277:3725-3734.

Bradbury, I. R., Hubert, S., Higgins, B., Bowman, S., Borza, T., Paterson, I. G., Snelgrove, P. V. R., Morris, C. J., Gregory, R. S., Hardie, D., Hutchings, J. A., Ruzzante, D. E., Taggart, C. T., and Bentzen, P. (2013). Genomic islands of divergence and their consequences for the resolution of spatial structure in an exploited marine fish. Evolutionary Applications, 6:450-461.

Carr, S. M., Kivlichan, D. G. S., Pepin, P., and Crutcher, D. C. (1999). Molecular phylogeny of Gadid fishes: Implications for the biogeographic origins of Pacific species. Canadian Journal of Zoology, 77:19-26.

Carr, S. M. and Marshall, H. D. (1991a). Detection of intraspecific DNA sequence variation in the mitochondrial cytochrome $b$ gene of Atlantic cod (Gadus morhua) by the polymerase chain reaction. Canadian Journal of Fisheries and Aquatic Sciences, 48:48-52.

Carr, S. M. and Marshall, H. D. (1991b). A direct approach to the measurement of genetic variation in fish populations: Applications of the polymerase chain reaction to studies of Atlantic cod (Gadus morhua). Journal of Fish Biology, 39(Supplement A):101-107.

Carr, S. M., Snellen, A. J., Howse, K. A., and Wroblewski, J. S. (1995). Mitochondrial DNA sequence variation and genetic stock structure of Atlantic cod (Gadus morhua) from bay and offshore locations on the Newfoundland continental shelf. Molecular Ecology, 4:79-88.

Case, R. A. J., Hutchinson, W. F., Hauser, L., Oosterhout, C. V., and Carvalho, G. R. (2005). Macro- and micro-geographic variation in pantophysin (PanI) allele frequencies in NE Atlantic cod Gadus morhua. Marine Ecology Progress Series, 301:267278.

Charif, D. and Lobry, J. (2007). SeqinR 1.0-2: a contributed package to the R project for statistical computing devoted to biological sequences retrieval and analysis. In Bastolla, U., Porto, M., Roman, H., and Vendruscolo, M., editors, Structural approaches to sequence evolution: Molecules, networks, populations, Biological and Medical Physics, Biomedical Engineering, pages 207-232. Springer Verlag, New York. ISBN : 978-3-540-35305-8.

Charlesworth, B., Charlesworth, D., and Barton, N. H. (2003). The effects of genetic and geographic structure on neutral variation. Annual Review of Ecology, Evolution and Systematics, 34:99-125.

Charlesworth, B., Nordborg, M., and Charlesworth, D. (1997). The effects of local selection, balanced polymorphism and background selection on equilibrium patterns of genetic diversity in subdivided populations. Genetical Research, 70:155-174.

Charlesworth, D. (2006). Balancing selection and its effects on sequences in nearby genome regions. PLoS Genetics, 2:e64. 
Coulson, M. W., Marshall, H. D., Pepin, P., and Carr, S. M. (2006). Mitochondrial genomics of gadine fishes: Implications for taxonomy and biogeographic origins from whole-genome data sets. Genome, 49:1115-1130.

Cruickshank, T. E. and Hahn, M. W. (2014). Reanalysis suggests that genomic islands of speciation are due to reduced diversity, not reduced gene flow. Molecular Ecology, 23:3133-3157.

Cunningham, K. M., Canino, M. F., Spies, I. B., and Hauser, L. (2009). Genetic isolation by distance and localized fjord population structure in Pacific cod (Gadus macrocephalus): Limited effective dispersal in the northeastern Pacific Ocean. Canadian Journal of Fisheries and Aquatic Sciences, 66:153-166.

Currat, M., Trabuchet, G., Rees, D., Perrin, P., Harding, R. M., Clegg, J. B., Langaney, A., and Excoffier, L. (2002). Molecular analysis of the $\beta$-globin gene cluster in the Niokholo Mandenka population reveals a recent origin of the $\beta^{\mathrm{S}}$ Senegal mutation. American Journal of Human Genetics, 70:207-223.

Donnelly, P. and Kurtz, T. G. (1999). Particle representations for measure-valued population models. Annals of Probability, 27:166-205.

Dray, S. and Dufour, A. (2007). The ade4 package: Implementing the duality diagram for ecologists. Journal of Statistical Software, 22:1-20.

Edgar, R. C. (2004). Muscle: Multiple sequence alignment with high accuracy and high throughput. Nucleic Acids Research, 32:1792-1797.

Eiríksson, G. M. and Árnason, E. (2013). Spatial and temporal microsatellite variation in spawning Atlantic cod, Gadus morhua, around Iceland. Canadian Journal of Fisheries and Aquatic Sciences, 70:1151-1158.

Eldon, B. (2011). Estimation of parameters in large offspring number models and ratios of coalescence times. Theoretical Population Biology, 80:16-28.

Eldon, B. (2012). Age of an allele and gene genealogies of nested subsamples for populations admitting large offspring numbers. arXiv, 1212.1792v1.

Eldon, B., Birkner, M., Blath, J., and Freund, F. (2015). Can the site-frequency spectrum distinguish exponential population growth from multiple-merger coalescents? Genetics, pages Early Online January 9, 2015.

Eldon, B. and Degnan, J. H. (2012). Multiple merger gene genealogies in two species: Monophyly, paraphyly, and polyphyly for two examples of lambda coalescents. Theoretical Population Biology, 82:117-130.

Eldon, B. and Wakeley, J. (2006). Coalescent processes when the distribution of offspring number among individuals is highly skewed. Genetics, 172:2621-2633.

Eldon, B. and Wakeley, J. (2008). Linkage disequilibrium under skewed offspring distribution among individuals in a population. Genetics, 178:1517-1532.

Eldon, B. and Wakeley, J. (2009). Coalescence times and $F_{S T}$ under a skewed offspring distribution among individuals in a population. Genetics, 181:615-629.

Ewing, B. and Green, P. (1998). Basecalling of automated sequencer traces using phred. II. error probabilities. Genome Research, 8:186-194.

Ewing, B., Hillier, L., Wendl, M., and Green, P. (1998). Base-calling of automated sequencer traces using phred. I. accuracy assessment. Genome Research, 8:175-185.

Fan, W., Kasahara, M., Gutknecht, J., Klein, D., Mayer, W. E., Jonker, M., and Klein, J. (1989). Shared class II MHC polymorphisms between humans and chimpanzees. Human Immunology, 26:107-121. 
Fevolden, S. E. and Pogson, G. H. (1995). Differences in nuclear DNA RFLPs between the Norwegian coastal and the Northeast Arctic populations of Atlantic cod. In Skjoldal, H. R., Hopkins, C., Eriksstad, K. E., and Leinaas, H. P., editors, Ecology of Fjords and Coastal Waters, pages 403-414, Amsterdam, The Netherlands. Elsevier Science Publishers.

Fevolden, S. E. and Pogson, G. H. (1997). Genetic divergence at the Synaptophysin (Syp I) locus among Norwegian coastal and north-east Arctic populations of Atlantic cod. Journal of Fish Biology, 51:895-908.

Foll, M. and Gaggiotti, O. (2008). A genome-scan method to identify selected loci appropriate for both dominant and codominant markers: A Bayesian perspective. Genetics, 180:977-993.

Gokcumen, O., Zhu, Q., Mulder, L. C. F., Iskow, R. C., Austermann, C., Scharer, C. D., Raj, T., Boss, J. M., Sunyaev, S., Price, A., Stranger, B., Simon, V., and Lee, C. (2013). Balancing selection on a regulatory region exhibiting ancient variation that predates Human-Neandertal divergence. PLoS Genetics, 9:e1003404.

Gordon, D., Abajian, C., and Green, P. (1998). Consed: A graphical tool for sequence finishing. Genome Research, 8:195-202.

Gouy, M., Guindon, S., and Gascuel, O. (2009). SeaView version 4: A multiplatform graphical user interface for sequence alignment and phylogenetic tree building. Molecular Biology and Evolution, 27:221-224.

Guinand, B., Lemaire, C., and Bonhomme, F. (2004). How to detect polymorphisms undergoing selection in marine fishes? a review of methods and case studies, including flatfishes. Journal of Sea Research, 51:167-182.

Guindon, S. and Gascuel, O. (2003). A simple, fast and accurate algorithm to estimate large phylogenies by maximum likelihood. Systematic Biology, 52:696-704.

Haldane, J. B. S. (1957). The cost of natural selection. Journal of Genetics, 55:511-524.

Halldórsdóttir, K. and Árnason, E. (2009a). Multiple linked $\beta$ and $\alpha$ globin genes in Atlantic cod: a PCR based strategy of genomic exploration. Marine Genomics, 2:169-181.

Halldórsdóttir, K. and Árnason, E. (2009b). Organization of a $\beta$ and $\alpha$ globin gene set in the teleost Atlantic cod, Gadus morhua. Biochemical Genetics, 47:817-830.

Harrang, E., Lapegue, S., Morga, B., and Bierne, N. (2013). A high load of nonneutral amino-acid polymorphisms explains high protein diversity despite moderate effective population size in a marine bivalve with sweepstakes reproduction. G3: Genes|Genomes|Genetics, 3(2):333-341.

Hedgecock, D. and Pudovkin, A. I. (2011). Sweepstakes reproductive success in highly fecund marine fish and shellfish: A review and commentary. Bulletin of Marine Science, 87:971-1002.

Hedrick, P. W. (1987). Genetic disequilibrium measures: Proceed with caution. Genetics, 117:331-341.

Hemmer-Hansen, J., Nielsen, E. E., Therkildsen, N. O., Taylor, M. I., Ogden, R., Geffen, A. J., Bekkevold, D., Helyar, S., Pampoulie, C., Johansen, T., Consortium, F., and Carvalho, G. R. (2013). A genomic island linked to ecotype divergence in Atlantic cod. Molecular Ecology, 22:2653-2667.

Hemmer-Hansen, J., Therkildsen, N. O., Meldrup, D., and Nielsen, E. E. (2014). Conserving marine biodiversity: Insights from life-history trait candidate genes in Atlantic 
cod (Gadus morhua). Conservation Genetics, 15:213-228.

Hubert, S., Higgins, B., Borza, T., and Bowman, S. (2010). Development of a SNP resource and a genetic linkage map for Atlantic cod (Gadus morhua). BMC Genomics, 11:191.

Hudson, R. R., Kreitman, M., and Aguadé, M. (1987). A test of neutral molecular evolution based on nucleotide data. Genetics, 116:153-159.

Johnson, M. S. and Wernham, J. (1999). Temporal variation of recruits as a basis of ephemeral genetic heterogeneity in the western rock lobster Panulirus cygnus. Marine Biology, 135:133-139.

Jombart, T. and Ahmed, I. (2011). Adegenet 1.3-1: New tools for the analysis of genome-wide SNP data. Bioinformatics, 27:3070-3071.

Joron, M., Frezal, L., Jones, R. T., Chamberlain, N. L., Lee, S. F., Haag, C. R., Whibley, A., Becuwe, M., Baxter, S. W., Ferguson, L., Wilkinson, P. A., Salazar, C., Davidson, C., Clark, R., Quail, M. A., Beasley, H., Glithero, R., Lloyd, C., Sims, S., Jones, M. C., Rogers, J., Jiggins, C. D., and ffrench Constant, R. H. (2011). Chromosomal rearrangements maintain a polymorphic supergene controlling butterfly mimicry. Nature, 477:203-206.

Kimura, M. (1983). The Neutral Theory of Molecular Evolution. Cambridge University Press, Cambridge.

Kingman, J. F. C. (1982a). The coalescent. Stochastic Processes and their Applications, 13:235-248.

Kingman, J. F. C. (1982b). On the genealogy of large populations. Journal of Applied Probability, 19A:27-43.

Lewontin, R. (1988). On measures of gametic disequilibrium. Genetics, 120:849-852.

Lurman, G. J., Koschnick, N., Pörtner, H.-O., and Lucassen, M. (2007). Molecular characterisation and expression of Atlantic cod (Gadus morhua) myoglobin from two populations held at two different acclimation temperatures. Comparative Biochemistry and Physiology Acta, 148:681-689.

Moen, T., Delghandi, M., Wesmajervi, M. S., Westgaard, J.-I., and Fjalestad, K. T. (2009). A snp/microsatellite genetic linkage map of the Atlantic cod (Gadus morhua). Animal Genetics, 40:993-996.

Moen, T., Hayes, B., Frank Nilsen and, M. D., Fjalestad, K. T., Fevolden, S., Berg, P. R., and Lien, S. (2008). Identification and characterisation of novel SNP markers in Atlantic cod: evidence for directional selection. BMC Genetics, 9:18.

Möhle, M. (1998). Robustness results for the coalescent. Journal of Applied Probability, 35:438-447.

Möhle, M. and Sagitov, S. (2001). A classification of coalescent processes for haploid exchangeable population models. Annals of Probability, 29:1547-1562.

Nei, M. and Hughes, A. L. (1991). Polymorphism and evolution of the major histocompatibility comples loci in mammals. In Selander, R., Clark, A., and Whittam, T., editors, Evolution at the Molecular Level, chapter 11, pages 222-247. Sinauer Associates, Inc., Sunderland, MA 01375.

Nielsen, E. E., Hansen, M. M., and Meldrup, D. (2006). Evidence of microsatellite hitch-hiking selection in Atlantic cod (Gadus morhua L.): Implications for inferring population structure in nonmodel organisms. Molecular Ecology, 15:3219-3229.

Nielsen, E. E., Hansen, M. M., Ruzzante, D. E., Meldrup, D., and Grønkjær (2003). 


\section{PeerJ Reviewing Manuscript}

Evidence of a hybrid-zone in Atlantic cod (Gadus morhua) in the Baltic and the Danish Belt Sea revealed by individual admixture analysis. Molecular Ecology, 12:1497-1508.

Nielsen, R. (2005). Molecular signatures of natural selection. Annual Review of Genetics, 39:197-218.

Ohashi, J., Naka, I., Patarapotikul, J., Hananantachai, H., Brittenham, G., Looareesuwan, S., Clark, A. G., , and Tokunaga, K. (2004). Extended linkage disequilibrium surrounding the Hemoglobin E variant due to malarial selection. American Journal of Human Genetics, 74:1198-1208.

Page, L. M., Espinosa-Pérez, H., Findley, L. T., Gilbert, C. R., Lea, R. N., Mandrak, N. E., Mayden, R. L., and Nelson, J. S. (2013). Common and Scientific Names of Fishes from the United States, Canada, and Mexico. Special Publication 34. American Fisheries Society, Bethesta, Maryland, 7th edition.

Pampoulie, C., Jakobsdóttir, K. B., Marteinsdóttir, G., and Thorsteinsson, V. (2007). Are vertical behaviour patterns related to the Pantophysin locus in the Atlantic cod (Gadus morhua L.)? Behavioral Genetics, 38:76-81.

Paradis, E. (2010). Pegas: an R package for population genetics with an integratedmodular approach. Bioinformatics, 26:419-420.

Paradis, E., Claude, J., and Strimmer, K. (2004). APE: Analyses of phylogenetics and evolution in R language. Bioinformatics, 20:289-290.

Pepin, P. and Carr, S. M. (1993). Morphological, meristic, and genetic analysis of stock structure in juvenile Atlantic cod (Gadus morhua) from the Newfoundland shelf. Canadian Journal of Fisheries and Aquatic Sciences, 50:1924-1933.

Pickrell1, J. K., Coop, G., Novembre, J., andJun Z. Li, S. K., Absher, D., Srinivasan, B. S., Barsh, G. S., Feldman, R. M. M. M. W., and Pritchard, J. K. (2009). Signals of recent positive selection in a worldwide sample of human populations. Genome Research, 19:922-933.

Pitman, J. (1999). Coalescents with multiple collisions. Annals of Probability, 27:18701902.

Pogson, G. H. (2001). Nucleotide polymorphism and natural selection at the Pantophysin (Pan I) locus in the Atlantic cod, Gadus morhua (L.). Genetics, 157:317-330.

Pogson, G. H. and Fevolden, S. (2003). Natural selection and the genetic differentiation of coastal and Arctic populations of the Atlantic cod in northern Norway: a test involving nucleotide sequence variation at the Pantophysin (PanI) locus. Molecular Ecology, 12:63-74.

Pogson, G. H. and Mesa, K. (2004). Positive Darwinian selection at the Pantophysin (Pan I) locus in marine Gadid fishes. Molecular Biology and Evolution, 21:65-75.

Poulsen, N. A., Hemmer-Hansen, J., Loeschcke, V., Carvalho, G. R., and Nielsen, E. E. (2011). Microgeographical population structure and adaptation in Atlantic cod Gadus morhua: spatio-temporal insights from gene-associated DNA markers. Marine Ecology Progress Series, 436:231-243.

R Core Team (2013). R A Language and Environment for Statistical Computing. R Foundation for Statistical Computing, Vienna, Austria.

Ranciaro, A., Campbell, M., Hirbo, J., Ko, W.-Y., Froment, A., Anagnostou, P., Kotze, M., Ibrahim, M., Nyambo, T., Omar, S., and Tishkoff, S. (2014). Genetic origins of lactase persistence and the spread of pastoralism in Africa. American Journal of 


\section{PeerJ Reviewing Manuscript}

Human Genetics, 94:496-510.

Rech, G. E., Sanz-Martin, J. M., Anisimova, M., Sukno, S. A., and Thon, M. R. (2014). Natural selection on coding and noncoding DNA sequences is associated with virulence genes in a plant pathogenic fungus. Genome Biology and Evolution, 6:2368-2379.

Rozas, J., Sánchez-DelBarrio, J. C., Messeguer, X., and Rozas, R. (2003). DnaSP, DNA polymorphism analyses by the coalescent and other methods. Bioinformatics, 19:2496-2497.

Sabeti, P. C., Varilly, P., Fry, B., Lohmueller, J., Hostetter, E., Cotsapas, C., Xie, X., Byrne, E. H., McCarroll, S. A., Gaudet, R., Schaffner, S. F., Lander, E. S., and Consortium, T. I. H. (2007). Genome-wide detection and characterization of positive selection in human populations. Nature, 449:913-919.

Sagitov, S. (1999). The general coalescent with asynchronous mergers of ancestral lines. Journal of Applied Probability, 36:1116-1125.

Sargsyan, O. and Wakeley, J. (2008). A coalescent process with simultaneous multiple mergers for approximating the gene genealogies of many marine organisms. Theoretical Population Biology, 74:104-114.

Sarvas, T. H. and Fevolden, S. E. (2005). Pantophysin (Pan I) locus divergence between inshore v. offshore and northern v. southern populations of Atlantic cod in the northeast Atlantic. Journal of Fish Biology, 67:444-469.

Schweinsberg, J. (2000). Coalescents with simultaneous multiple collisions. Electronic Journal of Probability, 5:1-50.

Schweinsberg, J. (2003). Coalescent processes obtained from supercritical GaltonWatson processes. Stochastic Processes and their Applications, 106:107-139.

Shin, J.-H., Blay, S., McNeney, B., and Graham, J. (2006). LDheatmap: An R function for graphical display of pairwise linkage disequilibria between single nucleotide polymorphisms. Journal of Statistical Software, 16:Code Snippet 3.

Sigurgíslason, H. and Árnason, E. (2003). Extent of mitochondrial DNA sequence variation in Atlantic cod from the Faroe Islands: A resolution of gene genealogy. Heredity, 91:557-564.

Star, B., Nederbragt, A. J., Jentoft, S., Grimholt, U., Malmstrom, M., Gregers, T. F., Rounge, T. B., Paulsen, J., Solbakken, M. H., Sharma, A., Wetten, O. F., Lanzen, A., Winer, R., Knight, J., Vogel, J.-H., Aken, B., Andersen, Ø., Lagesen, K., ToomingKlunderud, A., Edvardsen, R. B., Tina, K. G., Espelund, M., Nepal, C., Previti, C., Karlsen, B. O., Moum, T., Skage, M., Berg, P. R., Gjoen, T., Kuhl, H., Thorsen, J., Malde, K., Reinhardt, R., Du, L., Johansen, S. D., Searle, S., Lien, S., Nilsen, F., Jonassen, I., Omholt, S. W., Stenseth, N. C., and Jakobsen, K. S. (2011). The genome sequence of Atlantic cod reveals a unique immune system. Nature, 477:207-210.

Steinrücken, M., Birkner, M., and Blath, J. (2013). Analysis of DNA sequence variation within marine species using Beta-coalescents. Theoretical Population Biology, 87:1524.

Storz, J. F. (2005). Using genome scans of DNA polymorphisms to infer adaptive population divergence. Molecular Ecology, 14:671-688.

Storz, J. F., Payseur, B. A., and Nachman, M. W. (2004). Genome scans of DNA variability in humans reveal evidence for selective sweeps outside of Africa. Molecular Biology and Evolution, 21:1800-1811. 
Sun, C., Huo, D., Southard, C., Nemesure, B., Hennis, A., Leske, M. C., Wu, S.-Y., Witonsky, D. B., Olopade, O. I., and Rienzo, A. D. (2011). A signature of balancing selection in the region upstream to the human UGT2B4 gene and implications for breast cancer risk. Human Genetics, 130:767-775.

Tavaré, S. (2004). Ancestral inference in population genetics. In Picard, J., editor, Lectures on Probability Theory and Statistics. Ecole d'Eté de Probabilité de SaintFlour XXXI-2001, volume 1837 of Lecture Notes in Mathematics, pages 1-188. Springer Verlag, New York.

Tellier, A. and Lemaire, C. (2014). Coalescence 2.0: a multiple branching of recent theoretical developments and their applications. Molecular Ecology, 23:2637-2652.

Therkildsen, N. O., Hemmer-Hansen, J., Hedeholm, R. B., Wisz, M. S., Pampoulie, C., Meldrup, D., Bonanomi, S., Retzel, A., Olsen, S. M., , and Nielsen, E. E. (2013). Spatiotemporal SNP analysis reveals pronounced biocomplexity at the northern range margin of Atlantic cod Gadus morhua. Evolutionary Applications, 6:690-705.

Thompson, M. J. and Jiggins, C. D. (2014). Supergenes and their role in evolution. Heredity, 113:1-8.

Tishkoff, S. A., Reed, F. A., Ranciaro, A., Voight, B. F., Babbitt, C. C., Silverman, J. S., Powell, K., Mortensen, H. M., Hirbo, J. B., Osman, M., Ibrahim, M., Omar, S. A., Lema, G., Nyambo, T. B., Ghori, J., Bumpstead, S., Pritchard, J. K., Wray, G. A., and Deloukas, P. (2007). Convergent adaptation of human lactase persistence in Africa and Europe. Nature Genetics, 39:31-40.

Verrelli, B. C., McDonald, J. H., Argyropoulos, G., Destro-Bisol, G., Froment, A., Drousiotou, A., Lefranc, G., Helal, A. N., Loiselet, J., and Tishkoff, S. A. (2002). Evidence for balancing selection from nucleotide sequence analyses of human G6PD. American Journal of Human Genetics, 71:1112-1128.

Voight, B. F., Kudaravalli, S., Wen, X., and Pritchard, J. K. (2006). A map of recent positive selection in the human genome. PLoS Biology, 4:e72.

Wakeley, J. (2009). Coalescent Theory. Roberts and Company Publishers, Greenwood Village, Colorado, USA.

Wakeley, J. (2013). Coalescent theory has many new branches. Theoretical Population Biology, 87:1-4.

Wallimann, T., Tokarska-Schlattner, M., and Schlattner, U. (2011). The creatine kinase system and pleiotropic effects of creatine. Amino Acids, 40:1271-1296.

Wallimann, T., Wyss, M., Brdiczka, D., Nicolay, K., and Eppenberger, H. (1992). Intracellular compartmentation, structure and function of creatine kinase isoenzymes in tissues with high and fluctuating energy demands: the 'phosphocreatine circuit' for cellular energy homeostasis. Biochemical Journal, 281:21-40.

Williams, G. C. (1975). Sex and Evolution. Princeton University Press, Princeton, New Jersey.

Wilson, J. N., Rockett, K., Keating, B., Jallow, M., Pinder, M., Sisay-Joof, F., Newport, M., and Kwiatkowski, D. (2006). A hallmark of balancing selection is present at the promoter region of Interleukin 10. Genes \& Immunity, 7:680-683.

Wiuf, C. and Hein, J. (1999). Recombination as a point process along sequences.

Theoretical Population Biology, 55:248-259.

Wright, S. (1931). Evolution in Mendelian populations. Genetics, 16:97-159.

Wright, S. I. and Charlesworth, B. (2004). The HKA test revisited: A maximum- 


\section{PeerJ Reviewing Manuscript}

1137 likelihood-ratio test of the standard neutral model. Genetics, 168:1071-1076.

1138 Zhen, Y. and Andolfatto, P. (2012). Methods to detect selection on noncoding DNA. In

1139 Methods in Molecular Biology, pages 141-159. Springer Science Business Media. 


\section{PeerJ Reviewing Manuscript}

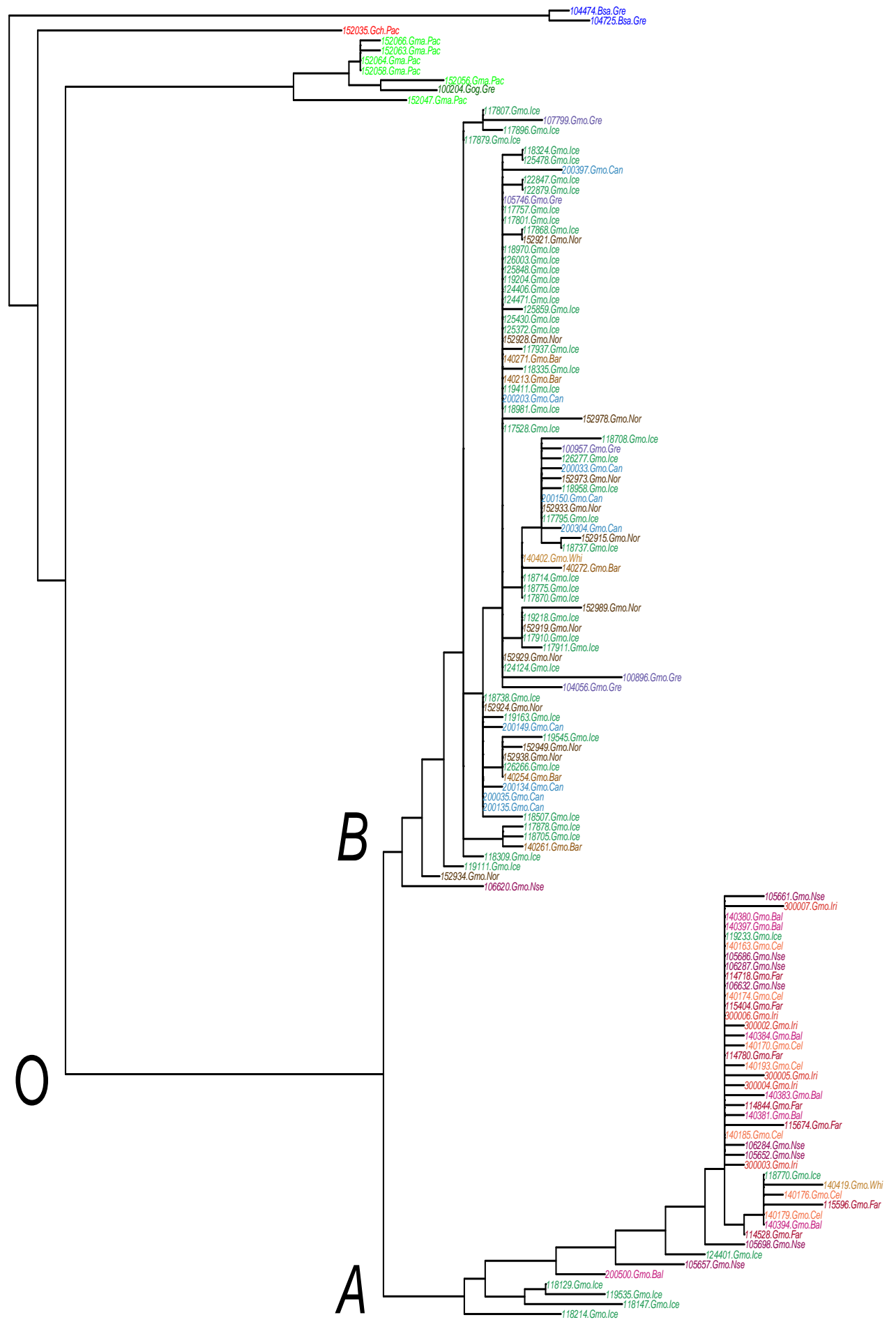

Figure 1. Maximum likelihood tree of Ckma variation ( $\boldsymbol{A}$ and $\boldsymbol{B}$ alleles) among 122 individual Atlantic cod and 10 individuals of four closely related outgroup $\mathbf{O}$ taxa, Boreogadus saida Bsa, Gadus chalcogramma Gch, Gadus macrocephalus Gma, and Gadus ogac Gog. Localities and color codes for Atlantic cod are the waters of Canada (Nova Scotia and Newfoundland) Can, Greenland Gre, Iceland Ice, Norway Nor, Faroe Islands Far, and from the Barents Sea Bar, White Sea Whi, North Sea Nor, Baltic Sea

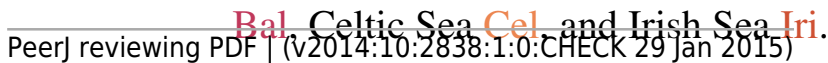




\section{PeerJ Reviewing Manuscript}
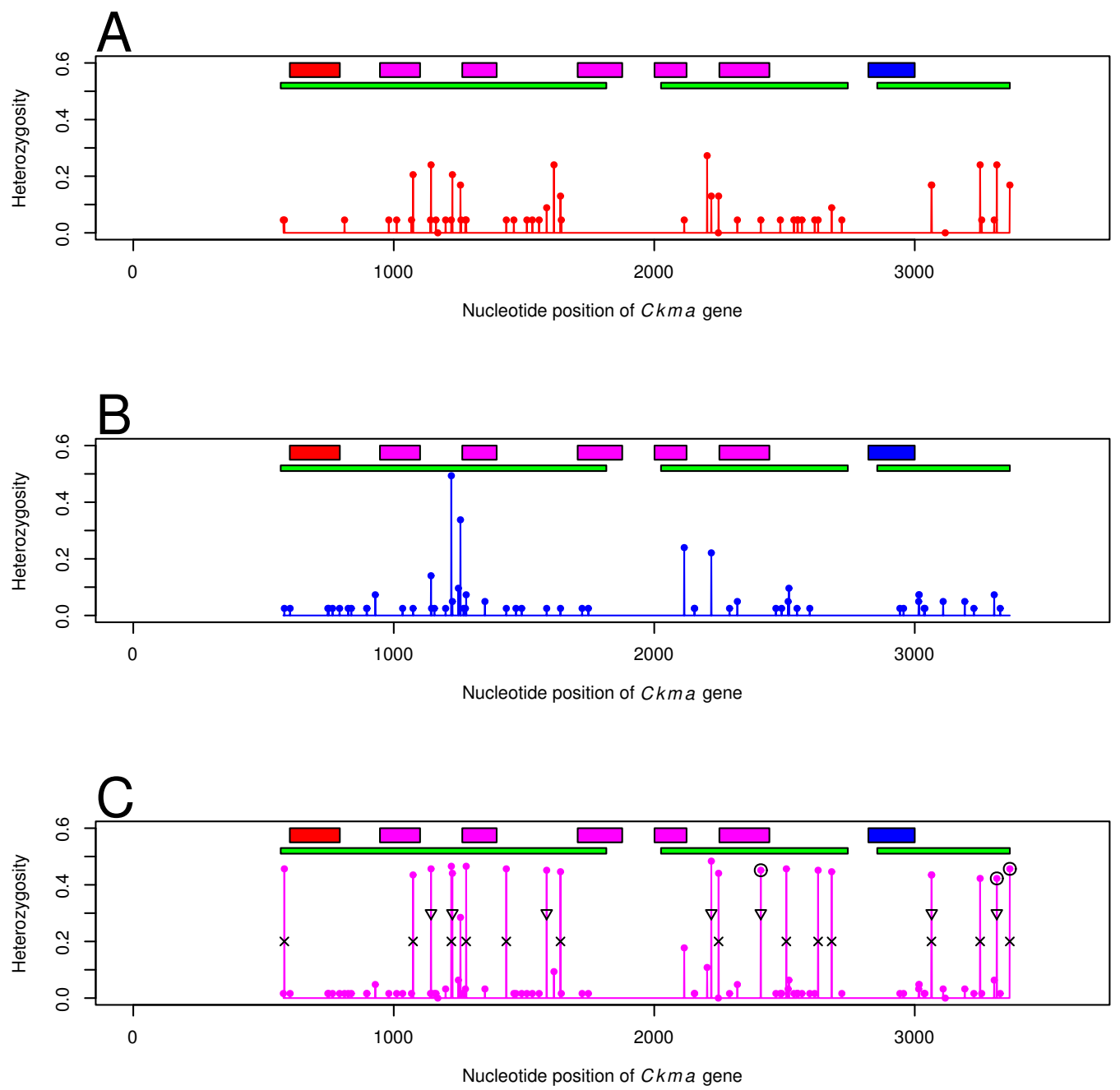

Figure 2. Heterozygosity per nucleotide site of $C \mathrm{kma}$ locus among $A$ alleles (red A panel, $n=43$ ), $B$ alleles (blue B panel, $n=79$ ), and all individuals combined (magenta C panel, $n=122$ ). Boxes represent exons, start (red), internal (magenta) and terminal (blue). Green boxes represent sequenced fragments trimmed to Phred score of at least 30. The black circles mark the three SNPs of Moen et al. (2008), Gm366-0514 locus with an $F_{S T}=0.83, G m 366-1022$ locus with an $F_{S T}=0.82$, and $G m 366-1073$ with an $F_{S T}=0.82$ from left to right respectively. Crosses mark mutant sites relative to outgroup that were fixed or nearly fixed among $A$ alleles. Triangles mark mutant sites relative to outgroup that have were fixed or nearly fixed among $B$ alleles. Gadus macrocephalus individual 152047 was used as the outgroup. 


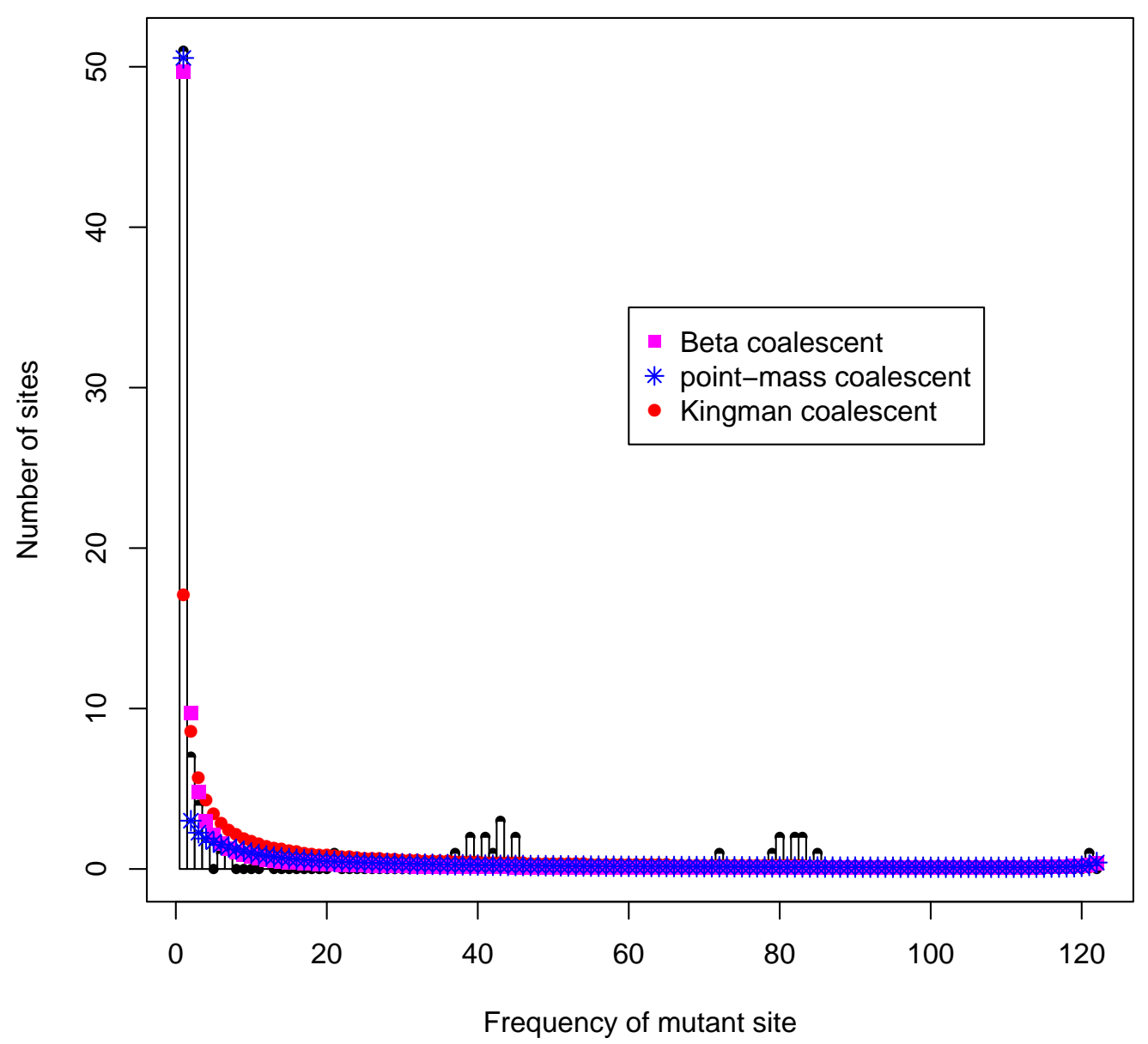

Figure 3. Unfolded site frequency spectrum of Atlantic cod Ckma gene. Gadus macrocephalus was used as the outgroup. Number of individuals $n=122$. Theroretical expectation under Kingman coalescent (red dots), $\operatorname{Beta}(2-\alpha, \alpha)$ coalescent (magenta squares), and point-mass coalescent (blue stars). 


\section{PeerJ Reviewing Manuscript}

Table 1. Summary statistics of polymorphism of $2500 \mathrm{bp}$ fragment of the $\mathrm{Ckma}$ gene, $711 \mathrm{bp}$ fragment of the $H b A 2$ gene and $1021 \mathrm{bp}$ fragment of the $M y g$ gene in Atlantic cod.

\begin{tabular}{lrrrrrrrr}
\hline Group & $n$ & $S$ & $H$ & $\hat{h}$ & $\hat{K}$ & $\hat{\theta}_{S}$ & $\hat{\pi}$ & $\hat{D}$ \\
\hline Ckma all & 122 & 87 & 72 & 0.959 & 10.62 & 0.0067 & 0.0043 & $-1.13^{\text {ns }}$ \\
Ckma North & 86 & 65 & 51 & 0.941 & 5.12 & 0.0054 & 0.0015 & $-1.97^{\text {ns }}$ \\
Ckma South & 36 & 45 & 23 & 0.891 & 3.61 & 0.0045 & 0.0015 & $-2.43^{* *}$ \\
Ckma A allele & 43 & 49 & 28 & 0.907 & 4.37 & 0.0047 & 0.0018 & $-2.20^{* *}$ \\
Ckma B allele & 79 & 53 & 44 & 0.930 & 3.10 & 0.0044 & 0.0013 & $-2.33^{* *}$ \\
\hline HbA2 all & 114 & 11 & 11 & 0.338 & 0.37 & 0.0030 & 0.0005 & $-2.09^{*}$ \\
HbA2 North & 95 & 9 & 9 & 0.347 & 0.39 & 0.0025 & 0.0005 & $-1.95^{*}$ \\
HbA2 South & 19 & 3 & 4 & 0.298 & 0.32 & 0.0016 & 0.0005 & $-0.95^{\text {ns }}$ \\
Myg all & 45 & 30 & 24 & 0.901 & 2.74 & 0.0071 & 0.0028 & $-2.03^{*}$ \\
Myg North & 36 & 28 & 20 & 0.894 & 2.65 & 0.0069 & 0.0027 & $-2.12^{*}$ \\
Myg South & 9 & 10 & 7 & 0.944 & 3.22 & 0.0037 & 0.0033 & $-0.58^{\text {ns }}$ \\
\hline
\end{tabular}

Sample size $n$, number of segregating sites $S$, number of haplotypes $H$, haplotype diversity $\hat{h}$, average number of pairwise differences $\hat{K}$, scaled population size from $S \theta_{S}$, nucleotide diversity $\hat{\pi}$, and Tajima's $\hat{D}$. ns is not significant, * represents $P<0.05$, and ${ }^{* *}$ represents $P<0.01$. 


\section{PeerJ Reviewing Manuscript}

Table 2. Parameter values minimizing the $\ell^{2}$ distance (sum of squares) between observed and expected unfolded site frequency spectra for nuclear genes and for mtDNA variation of various localities.

\begin{tabular}{lcccccrl}
\hline Source & $\hat{\alpha}$ & $\hat{\psi}$ & $\ell^{2}(\hat{\alpha})$ & $\ell^{2}(\hat{\psi})$ & $\ell^{2}(0)$ & $n$ & Reference \\
\hline Nuclear locus & & & & & & & \\
Hba2 & 1.000 & 0.230 & 0.035 & 0.016 & 0.431 & 113 & This study \\
Myg & 1.000 & 0.225 & 0.010 & 0.018 & 0.230 & 45 & This study \\
Ckma & 1.280 & 0.070 & 0.006 & 0.007 & 0.141 & 122 & This study \\
Ckma $^{A}$ & 1.100 & 0.170 & 0.017 & 0.012 & 0.161 & 43 & This study \\
Ckma $^{B}$ & 1.140 & 0.120 & 0.006 & 0.015 & 0.189 & 79 & This study \\
\hline Locality for mtDNA & & & & & & & \\
Newfoundland & 1.550 & 0.015 & 0.014 & 0.028 & 0.084 & 378 & Carr et al. \\
Greenland & 1.945 & 0.005 & 0.072 & 0.071 & 0.072 & 78 & Árnason et al. (2000) \\
Iceland & 1.550 & 0.010 & 0.006 & 0.050 & 0.078 & 519 & Árnason et al. (2000) \\
Norway & 1.895 & 0.015 & 0.093 & 0.089 & 0.095 & 100 & AP 1996 \\
White Sea & 2.000 & 0.005 & 0.551 & 0.554 & 0.551 & 109 & Árnason et al. (1998) \\
Faroe Islands & 1.555 & 0.050 & 0.059 & 0.055 & 0.093 & 74 & SA 2003 \\
Baltic Sea & 2.000 & 0.005 & 0.105 & 0.109 & 0.105 & 109 & Árnason et al. (1998) \\
Atlantic & 1.530 & 0.010 & 0.006 & 0.055 & 0.249 & 1278 & Árnason (2004) \\
\hline
\end{tabular}

Based on method of Birkner et al. (2013b). Parameters $\alpha$ of the Beta $(2-\alpha, \alpha)$, and $\psi$ of the point-mass coalescent and their respective $\ell^{2}$. The $\ell^{2}(0)$ is based on the Kingman coalescent for which $\alpha=2$. For the mtDNA Carr et al. refers to Carr and Marshall (1991a,b); Carr et al. (1995); Pepin and Carr (1993), AP 1996 refers to Árnason and Pálsson (1996), and SA 2003 refers to Sigurgíslason and Árnason (2003). 
1

Maximum likelihood tree of $C k m a$ variation ( $A$ and $B$ alleles) among 122 individual Atlantic cod and 10 individuals of four closely related outgroup $O$ taxa, Boreogadus saida Bsa, Gadus chalcogramma Gch, Gadus macrocephalus 


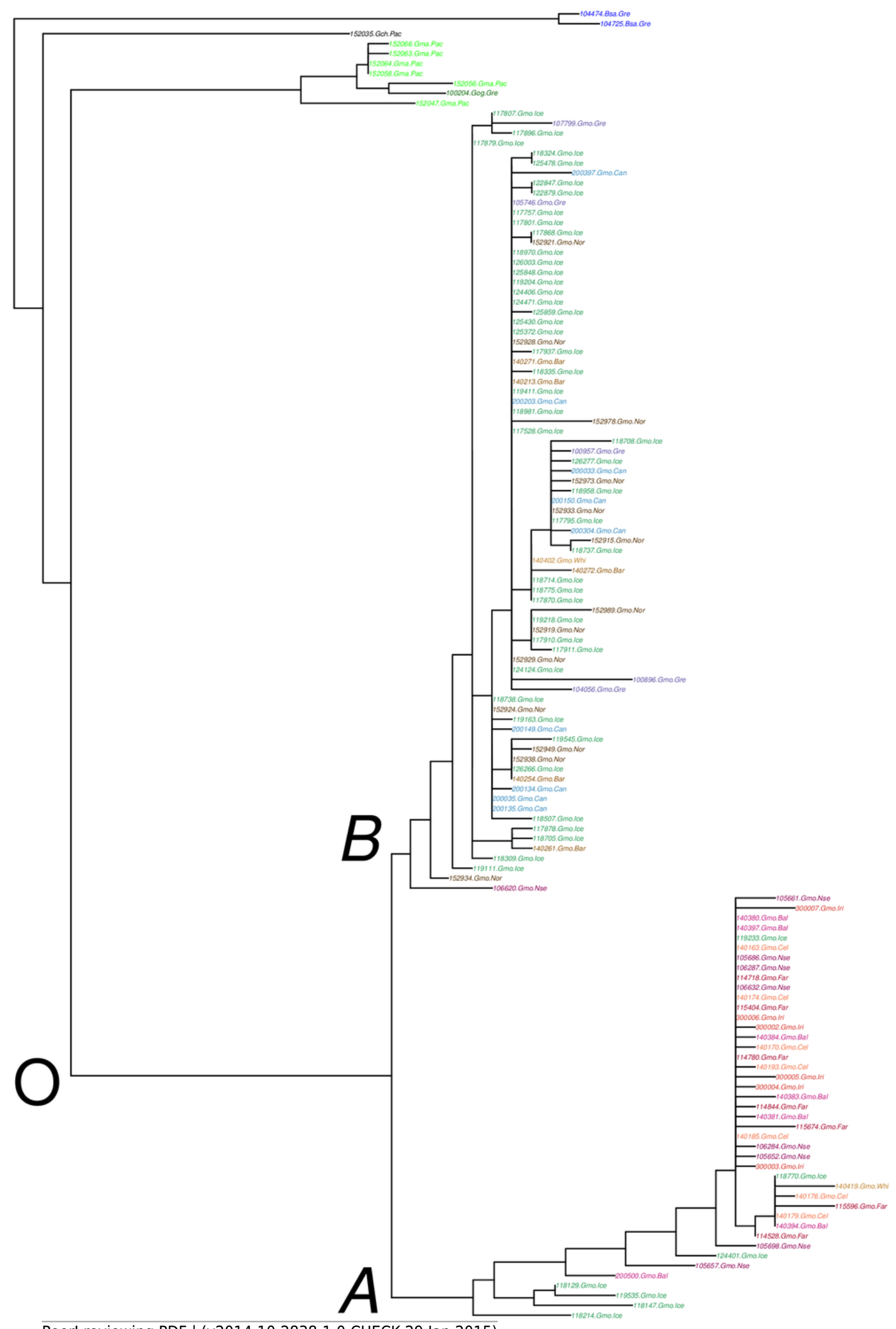


2

Heterozygosity per nucleotide site of $C k m a$ locus among $A$ alleles (red A panel, $n=43$ ), $B$ alleles (blue $B$ panel, $n=79$ ), and all individuals combined (magenta $C$ panel, $n=$ 122).

Boxes represent exons, start (red), internal (magenta) and terminal (blue). Green boxes represent sequenced fragments trimmed to Phred score of at least 30. The black circles mark the three SNPs of Moen et al. (2008), Gm366-0514 locus with an $F_{S T}=0.83, G m 366-1022$ locus with an $F_{S T}=0.82$, and $G m 366-1073$ with an $F_{S T}=0.82$ from left to right respectively. Crosses mark mutant sites relative to outgroup that were fixed or nearly fixed among $A$ alleles. Triangles mark mutant sites relative to outgroup that were fixed or nearly fixed among $B$ alleles. Gadus macrocephalus individual 152047 was used as the outgroup. 

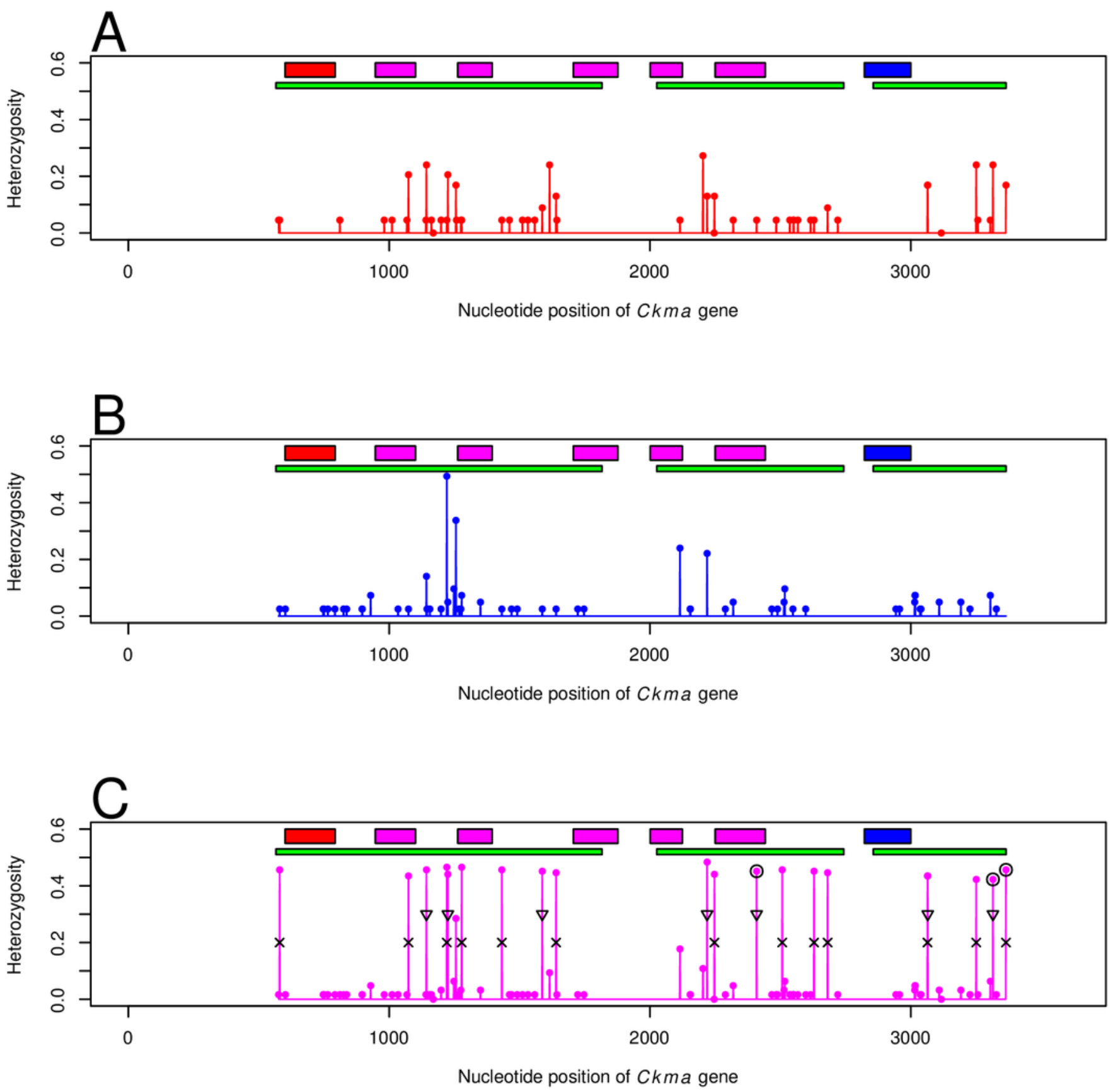


\section{3}

Unfolded site frequency spectrum of Atlantic cod Ckma gene.

Gadus macrocephalus was used as the outgroup. Number of individuals $n=122$. Theroretical expectation under Kingman coalescent (red dots), Beta $(2-\alpha, \alpha)$ coalescent (magenta squares), and point-mass coalescent (blue stars).

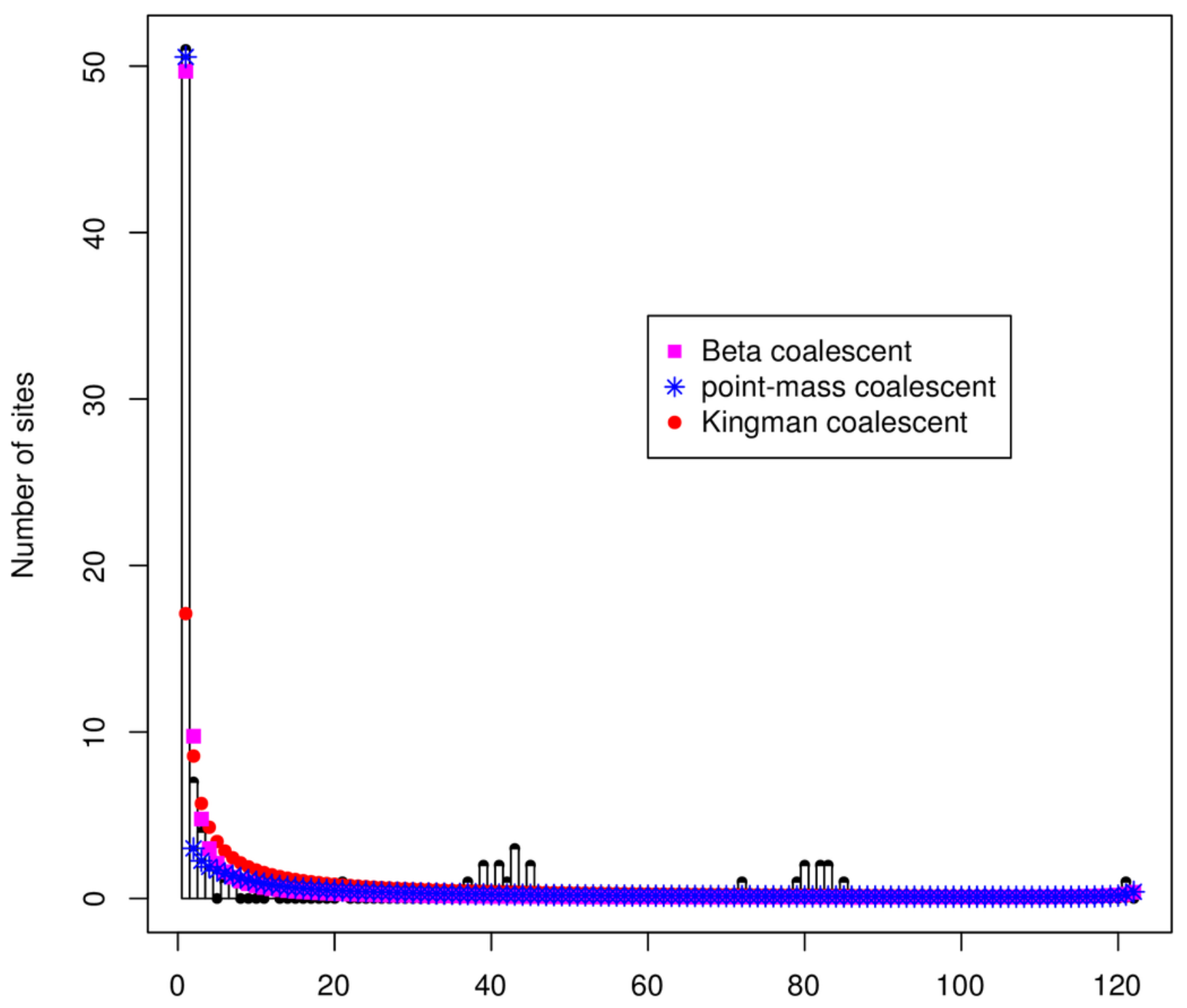

Frequency of mutant site 


\section{Table $\mathbf{1}_{\text {(on next page) }}$}

Summary statistics. of polymorphism of $2500 \mathrm{bp}$ fragment of the Ckma gene, $711 \mathrm{bp}$ fragmen $H b A 2$ gene and 1021 bp fragment of the Myg gene in Atlantic cod.

Sample size $n$, number of segregating sites $S$, number of haplotypes $H$, haplotype diversity $h$, average number of pairwise differences $K$, scaled population size from $S \theta_{s}$, nucleotide diversity $\pi$, and Tajima's $D$. ns is not significant, * represents $P<0.05$, and ** represents $P<$ 0.01 . 


\section{PeerJ Reviewing Manuscript}

Table 1. Summary statistics of polymorphism of $2500 \mathrm{bp}$ fragment of the $\mathrm{Ckma}$ gene, $711 \mathrm{bp}$ fragment of the $H b A 2$ gene and $1021 \mathrm{bp}$ fragment of the $M y g$ gene in Atlantic cod.

\begin{tabular}{lrrrrrrrr}
\hline Group & $n$ & $S$ & $H$ & $\hat{h}$ & $\hat{K}$ & $\hat{\theta}_{S}$ & $\hat{\pi}$ & $\hat{D}$ \\
\hline Ckma all & 122 & 87 & 72 & 0.959 & 10.62 & 0.0067 & 0.0043 & $-1.13^{\text {ns }}$ \\
Ckma North & 86 & 65 & 51 & 0.941 & 5.12 & 0.0054 & 0.0015 & $-1.97^{\text {ns }}$ \\
Ckma South & 36 & 45 & 23 & 0.891 & 3.61 & 0.0045 & 0.0015 & $-2.43^{* *}$ \\
Ckma A allele & 43 & 49 & 28 & 0.907 & 4.37 & 0.0047 & 0.0018 & $-2.20^{* *}$ \\
Ckma B allele & 79 & 53 & 44 & 0.930 & 3.10 & 0.0044 & 0.0013 & $-2.33^{* *}$ \\
\hline HbA2 all & 114 & 11 & 11 & 0.338 & 0.37 & 0.0030 & 0.0005 & $-2.09^{*}$ \\
HbA2 North & 95 & 9 & 9 & 0.347 & 0.39 & 0.0025 & 0.0005 & $-1.95^{*}$ \\
HbA2 South & 19 & 3 & 4 & 0.298 & 0.32 & 0.0016 & 0.0005 & $-0.95^{\text {ns }}$ \\
Myg all & 45 & 30 & 24 & 0.901 & 2.74 & 0.0071 & 0.0028 & $-2.03^{*}$ \\
Myg North & 36 & 28 & 20 & 0.894 & 2.65 & 0.0069 & 0.0027 & $-2.12^{*}$ \\
Myg South & 9 & 10 & 7 & 0.944 & 3.22 & 0.0037 & 0.0033 & $-0.58^{\text {ns }}$ \\
\hline
\end{tabular}

Sample size $n$, number of segregating sites $S$, number of haplotypes $H$, haplotype diversity $\hat{h}$, average number of pairwise differences $\hat{K}$, scaled population size from $S \theta_{S}$, nucleotide diversity $\hat{\pi}$, and Tajima's $\hat{D}$. ns is not significant, * represents $P<0.05$, and ${ }^{* *}$ represents $P<0.01$. 
Table 2 (on next page)

Parameter values minimizing the $\ell^{2}$ distance (sum of squares) between observed and expected unfolded site frequency spectra for nuclear genes and for mtDNA variation of various localities.

Based on method of Birkner et al. (2013b). Parameters $\alpha$ of the Beta $(2-\alpha, \alpha)$, and $\psi$ of the point-mass coalescent and their respective $\ell^{2}$. The $\ell^{2}(0)$ is based on the Kingman coalescent for which $\alpha=2$. For the mtDNA Carr et al. refers to Carr and Marshall (1991a,b); Carr et al. (1995); Pepin and Carr (1993), AP 1996 refers to Árnason and Pálsson (1996), and SA 2003 refers to Sigurgíslason and Árnason (2003). 


\section{PeerJ Reviewing Manuscript}

Table 2. Parameter values minimizing the $\ell^{2}$ distance (sum of squares) between observed and expected unfolded site frequency spectra for nuclear genes and for mtDNA variation of various localities.

\begin{tabular}{lcccccrl}
\hline Source & $\hat{\alpha}$ & $\hat{\psi}$ & $\ell^{2}(\hat{\alpha})$ & $\ell^{2}(\hat{\psi})$ & $\ell^{2}(0)$ & $n$ & Reference \\
\hline Nuclear locus & & & & & & & \\
Hba2 & 1.000 & 0.230 & 0.035 & 0.016 & 0.431 & 113 & This study \\
Myg & 1.000 & 0.225 & 0.010 & 0.018 & 0.230 & 45 & This study \\
Ckma & 1.280 & 0.070 & 0.006 & 0.007 & 0.141 & 122 & This study \\
Ckma $^{A}$ & 1.100 & 0.170 & 0.017 & 0.012 & 0.161 & 43 & This study \\
Ckma $^{B}$ & 1.140 & 0.120 & 0.006 & 0.015 & 0.189 & 79 & This study \\
\hline Locality for mtDNA & & & & & & & \\
Newfoundland & 1.550 & 0.015 & 0.014 & 0.028 & 0.084 & 378 & Carr et al. \\
Greenland & 1.945 & 0.005 & 0.072 & 0.071 & 0.072 & 78 & Árnason et al. (2000) \\
Iceland & 1.550 & 0.010 & 0.006 & 0.050 & 0.078 & 519 & Árnason et al. (2000) \\
Norway & 1.895 & 0.015 & 0.093 & 0.089 & 0.095 & 100 & AP 1996 \\
White Sea & 2.000 & 0.005 & 0.551 & 0.554 & 0.551 & 109 & Árnason et al. (1998) \\
Faroe Islands & 1.555 & 0.050 & 0.059 & 0.055 & 0.093 & 74 & SA 2003 \\
Baltic Sea & 2.000 & 0.005 & 0.105 & 0.109 & 0.105 & 109 & Árnason et al. (1998) \\
Atlantic & 1.530 & 0.010 & 0.006 & 0.055 & 0.249 & 1278 & Árnason (2004) \\
\hline
\end{tabular}

Based on method of Birkner et al. (2013b). Parameters $\alpha$ of the Beta $(2-\alpha, \alpha)$, and $\psi$ of the point-mass coalescent and their respective $\ell^{2}$. The $\ell^{2}(0)$ is based on the Kingman coalescent for which $\alpha=2$. For the mtDNA Carr et al. refers to Carr and Marshall (1991a,b); Carr et al. (1995); Pepin and Carr (1993), AP 1996 refers to Árnason and Pálsson (1996), and SA 2003 refers to Sigurgíslason and Árnason (2003). 